Review Article

\title{
Review on the Antibacterial Mechanism of Plant-Derived Compounds against Multidrug-Resistant Bacteria (MDR)
}

\author{
Najwan Jubair (D), Mogana Rajagopal (D), Sasikala Chinnappan ${ }^{1}{ }^{1}$, \\ Norhayati Binti Abdullah $\mathbb{D}^{2},{ }^{2}$ and Ayesha Fatima $\mathbb{D}^{3}$ \\ ${ }^{1}$ Faculty of Pharmaceutical Sciences, UCSI University, Kuala Lumpur 56000, Malaysia \\ ${ }^{2}$ Forest Research Institute Malaysia (FRIM), Kuala Lumpur 52109, Selangor, Malaysia \\ ${ }^{3}$ Beykoz Institute of Life Sciences and Biotechnology, Bezmialem Vakif University, Istanbul, Turkey \\ Correspondence should be addressed to Najwan Jubair; 1001953426@ucsiuniversity.edu.my
}

Received 6 April 2021; Revised 27 June 2021; Accepted 24 July 2021; Published 17 August 2021

Academic Editor: Armando Zarrelli

Copyright (C) 2021 Najwan Jubair et al. This is an open access article distributed under the Creative Commons Attribution License, which permits unrestricted use, distribution, and reproduction in any medium, provided the original work is properly cited.

Microbial resistance has progressed rapidly and is becoming the leading cause of death globally. The spread of antibiotic-resistant microorganisms has been a significant threat to the successful therapy against microbial infections. Scientists have become more concerned about the possibility of a return to the pre-antibiotic era. Thus, searching for alternatives to fight microorganisms has become a necessity. Some bacteria are naturally resistant to antibiotics, while others acquire resistance mainly by the misuse of antibiotics and the emergence of new resistant variants through mutation. Since ancient times, plants represent the leading source of drugs and alternative medicine for fighting against diseases. Plants are rich sources of valuable secondary metabolites, such as alkaloids, quinones, tannins, terpenoids, flavonoids, and polyphenols. Many studies focus on plant secondary metabolites as a potential source for antibiotic discovery. They have the required structural properties and can act by different mechanisms. This review analyses the antibiotic resistance strategies produced by multidrug-resistant bacteria and explores the phytochemicals from different classes with documented antimicrobial action against resistant bacteria, either alone or in combination with traditional antibiotics.

\section{Introduction}

Bacterial infection is considered as one of the significant contributors to human illness in developed and developing countries [1]. Pathogens evolve with time and gain resistance to formerly discovered antibiotics. Among two million people infected each year worldwide with various types of bacteria, 700 thousands of them died because of bacterial resistance $[2,3]$. For instance, methicillin-resistant Staphylococcus aureus accounts for 50,000 deaths annually in the United States and Europe [4]. Simultaneously, antibioticresistant Mycobacterium tuberculosis infected about 480,000 people in underdeveloped countries during 2013 [4].

According to the CDC's antibiotic-resistance threat report in 2019, over 2.8 million antibiotic-resistance infections were recorded in the United States [5]. Carbapenemresistant Acinetobacter seems to be the top, urgent threat, with 700 estimated deaths in the United States in 2017 [5]. This number is estimated to increase to ten million cases by 2050 due to lack of response to medication despite all efforts and costs to overcome this issue, which is estimated to be $\$ 100.2$ trillion by 2050 [6]. Some bacteria are innately resistant to antibiotics, while others acquired resistance with time. According to the European Center for Disease Prevention and Control (ECDC), Center for Disease Prevention and Control (CDC), and Infectious Diseases Society of America (IDSA) [7], acquired bacterial resistance can be categorized into three main classes: extensive-drug resistance (XDR), pan drug resistance (PDR), and multidrug resistance (MDR) [8].

Extensive-drug resistance (XDR) is defined as nonsusceptibility to at least one antibiotic in all but two or fewer antimicrobial classes, which means that bacterial strains remain sensitive to only one or two antibiotic categories [9]. 
An example of this class is XDR-M. tuberculosis, which resists isoniazid, rifampicin, fluoroquinolones, and at least one agent of the three second-line treatments (amikacin, kanamycin, or capreomycin) [10]. For pan drug resistance (PDR), the name derives from the Greek word "pan," which means "all," referring to bacterial resistance to all commercially available or routinely tested antimicrobial agents [8].

Multidrug-resistant microorganisms (MDR) assign bacteria resistant to a minimum of one agent in three or more antimicrobial classes [7]. The most common types of MDR bacteria are methicillin-resistant $S$. aureus (MRSA), vancomycin-resistant Enterococci (VRE), carbapenem-resistant A. baumannii (CRAB), multidrug-resistant $P$. aeruginosa, Enterobacteriaceae that produces extendedspectrum $\beta$-lactamases, and carbapenem-resistant Enterobacteriaceae (CRE) [11]. These resistant strains are considered a major threat to human health as the outcome associated with MDR bacteria is worse than patients infected with more susceptible organisms, and the rising cost associated with these infections [11].

Consequently, scientists have become more concerned about the efficacy of current antibiotics against various types of infectious diseases. Many studies concentrated on finding new alternative medication as a way to overcome resistant problems. Nature represents a potential source for drug discovery. For decades, plants' phytochemicals attracted scientists as they are numerous in structure, have fewer side effects, and are more acceptable by people $[12,13]$. Most of these phytochemicals have antimicrobial activity $[12,14]$. Approximately, 25\% of the current pharmacopeia is from plant-derived compounds [15].

About $80 \%$ of the developing countries depend on plantderived medicines as their first-line treatment [16]. Moreover, plants remain the core of several medical practices such as Ayurveda (traditional Indian medicinal systems) and Traditional Chinese medicine (TCM) [17]. For instance, Oxalis corniculata extract has intense antimicrobial activity against E. coli, MDR-Salmonella typhi, K. pneumoniae, and Citrobacter koseri (inhibition zones are $17 \mathrm{~mm}, 13 \mathrm{~mm}$, $16 \mathrm{~mm}, 11 \mathrm{~mm}$, and $12 \mathrm{~mm}$, respectively) [18].

Five local plants that have been used by indigenous people in Iraq were tested for their antimicrobial effect toward S. epidermidis and K. pneumoniae [19]. It was shown that the type of solvents and the extract concentration markedly affect their activity, with Punica granatum L. being the most active extract regardless of the solvent type [19]. Ludwigia parviflora Roxb. plant has been used traditionally in India for wound dressing and as a remedy for dysentery [20]. The ethanolic fruit extract of this plant showed significant antibacterial activity toward E. coli, B. subtilis, S. typhi, and S. pyogenes in a study that emphasizes the use of this plant traditionally to treat various diseases [20]. The present review focused on describing the bacterial mechanism of resistance, and providing an updated review of plant phytochemicals' antibacterial mechanism against multidrug-resistant bacteria.

\section{Mechanism of Bacterial Resistance}

About 20,000 resistant genes have been identified from bacteria, and various mechanisms have been highlighted to explain bacterial resistance to traditional antimicrobial agents [21]. The first case of antibiotic resistance was detected during the 1950s with Salmonella, Shigella, and E. coli species [22]. However, it took two decades to recognize this rising problem, as in the 1970s, several cases were reported with resistance to penicillin, tetracyclines, and chloramphenicol [22]. The proposed mechanisms for bacterial resistance were rarely tested on a clinical basis [23]. It is ambiguous whether each microorganism has its mechanism, or there is a similar pathway shared by several bacteria to produce resistance [23].

Some bacteria are naturally resistant to antibiotics, while others acquire resistance mainly through the misuse of antibiotics and the emergence of new resistant strains [24]. The mechanism of antibacterial resistance ranges from accelerating antibiotics efflux through bacterial efflux pumps so as to decrease the time required for medication to diffuse inside bacteria [25], alteration of bacterial porins' structure which decreases bacterial permeability to antibiotic influx [26], destruction of antibacterial agents by hydrolytic enzymes [25], to alteration of binding sites for antibiotics [27]. For bacteria, they may fight antibiotics by one mechanism or by combining more than one to produce their resistance [25]. Understanding resistance mechanism is essential to identify the possible target for effective medication in the future. Each of the mechanisms mentioned above will be discussed in detail as follows.

2.1. Efflux Pump. To produce antibacterial activity, the antibiotic needs to enter the bacterial cell at adequate concentration and stay for a considerable time to perform its action [25]. Many MDR bacteria counter antibiotics by the efflux pump mechanism. Efflux pumps are proteinaceous transporters located in the cytoplasmic membrane that function as regulators of the bacterial internal environment [28]. They aid bacteria in removing toxins as well as antibiotics. For the first time, scientists identified the efflux pump as the cause of E. coli resistance to tetracycline [29]. However, it was recently considered a major cause of resistance [28].

Based on sequence similarities, source of energy, substrate binding, and the number of components, bacterial efflux transporters are classified into five prominent families [28]; Resistance-nodulation division (RND), which is a specific group for Gram-negative bacteria [30], adenosine triphosphate (ATP)-binding cassette superfamily (ABC) [31], multidrug and toxic compound extrusion (MATE) [32], major facilitator superfamily (MFS) [33], and small multidrug resistance family (SMR) [34]. Figure 1 is adopted from Blanco et al., presenting the major efflux transporters in bacteria [28]. $\mathrm{ABC}$, MATE, MFS, and SMR distribute to a large degree in Gram-positive and Gram-negative bacteria [28]. 


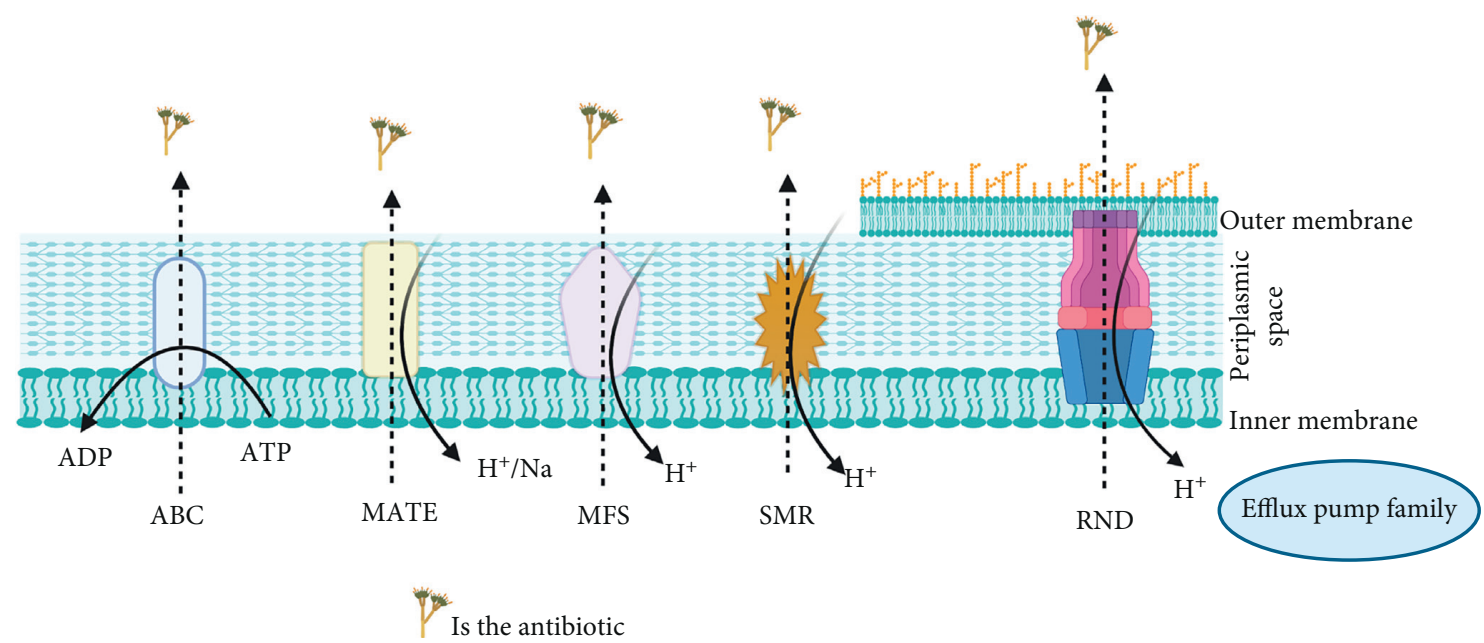

FIGURE 1: Schematic representation showing the major types of efflux pump families present in bacteria. Presented are resistance-nodulation family (RND) which is specific for Gram-negative bacteria; small multidrug resistance (SMR) locates in the inner membrane; major facilitator superfamily (MFS), multidrug and toxin extrusion (MATE), and adenosine triphosphate-binding cassette (ABC) superfamily are located in the inner membrane. All efflux pumps regulate toxins and antibiotics transports in energy-dependent manner.

Through efflux pumps, bacteria extrude antimicrobial agents faster than usual. Thus, the agent's time to reach the target site at a considerable concentration will decrease [25]. Bacterial efflux pumps are encoded by genes located either in mobile genetic elements (MGEs), representing genetic materials like plasmids and transposons found in all organisms, including humans or in chromosomes [35]. Recently, about 20 genes encoded in bacterial efflux have been identified, most of them located in MGEs and modulate bacterial resistance of most Gram-positive bacteria [28]. Various MDR efflux pumps in Enterobacteriaceae and $P$. aeruginosa belong to the RND family, and effectively extrude tetracyclines [36].

Due to RND pumps, resistance also occurs in many antibiotics like chloramphenicol, beta-lactams, fluoroquinolones, and fusidic acid [28]. S. pyogenes, S. pneumoniae, Streptococci, and some Gram-positive bacteria resist macrolides by efflux pumps mainly through the $\mathrm{ABC}$ family present in the chromosome [37]. This mechanism of resistance was initially recognized in S. epidermidis [37]. Antibiotic resistance through efflux pump mechanism also manifests in A. baumannii by which AdeABC and RND efflux pumps release antibiotics like aminoglycosides, tigecycline, lactams, chloramphenicol, erythromycin, and tetracycline from the cell [38], which results in a reduction of drug accumulation at the target site and increases the minimum inhibitory concentration of antibiotics making them ineffective [38].

2.2. Alteration of Membrane Permeability. Gram-negative bacteria are characterized by the presence of an outer membrane $(\mathrm{OM})$, which functions as an additional protection layer against harmful compounds [39]. Porins are proteins present in the bacterial outer membrane that can form water-filled holes controlling the passage of several materials and nutrients through this membrane [25]. They represent one of the possible targets for antibacterial agents
[25]. Porins were first identified in E. coli (Figure 2 adopted from Katherine Phan and Thomas Ferenci illustration) [40]. They can be classified based on activity into specific and nonspecific [41]. Specific porins are more selective for certain compounds such as Lam B, and selectively uptake maltose, maltodextrin, and Fep A specific for iron complex [42]. In contrast, nonspecific or general porins are involved in membrane permeability and linked to bacterial resistance to the antimicrobial agents [42].

Based on structure, porins are divided into monomeric, dimeric, and trimeric [39]. Besides, they can be classified according to their role in antibiotic transport and membrane integrity into three groups; specific porins for antibiotic transport (LamB, YddB), porin specific for membrane integrity $(\mathrm{OmpA})$, and nonspecific porins act on both antibiotic transport and membrane safety $(\mathrm{OmpC}, \mathrm{OmpF})$ [39]. Alteration of the porin structure is associated with an increase in bacterial resistance to antibiotics [25]. For example, OmpF porin is responsible for the transportation of several antibiotics such as $\beta$-lactams and fluoroquinolones.

Mutation in this porin renders resistance in E. coli [43], K. pneumoniae [44], and P. aeruginosa [45]. A. baumannii resists carbapenem by reducing porin expressions such as Caro and Omp 22-33. Besides, it changes the outer membrane integrity to acquire colistin resistance [46]. The mechanism by which $P$. aeruginosa resists various types of antibiotics is classified into intrinsic, acquired, and adaptive [47]. Alteration of the outer-membrane main component, the liposaccharides (LPs), is an example of intrinsic-type resistance by which $P$. aeruginosa fights antibiotics like $\beta$-lactams, quinolones, and aminoglycosides [47].

2.3. Destruction of Antibiotics. Bacterial enzymes (Figure 3, adopted from Egorov et al. [48] with slight modifications) are a group of enzymes associated with the biosynthesis of the bacterial cell wall, nucleic acids, and metabolites synthesis. Some antimicrobial agents directly target these 


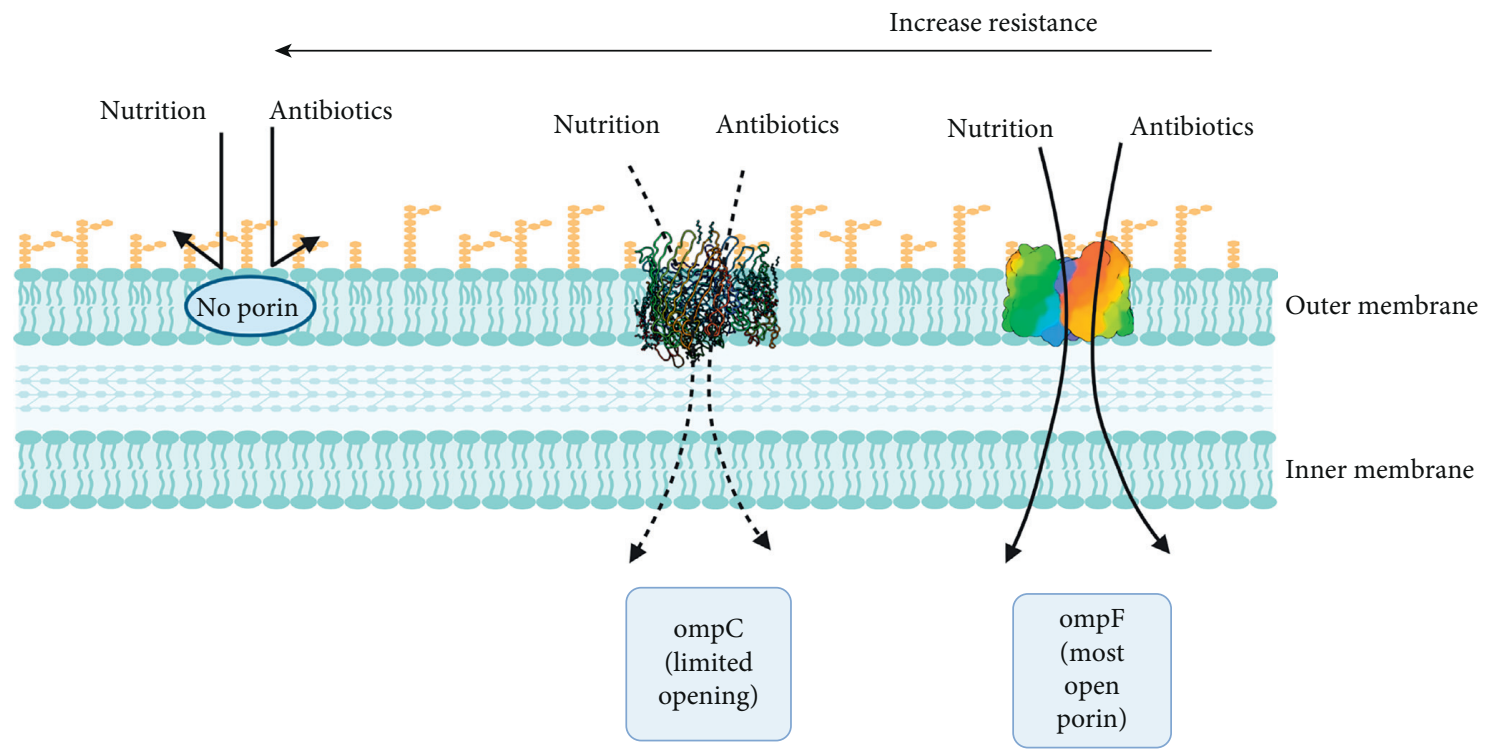

FIgURE 2: Effect of porins in membrane permeability of E. coli.

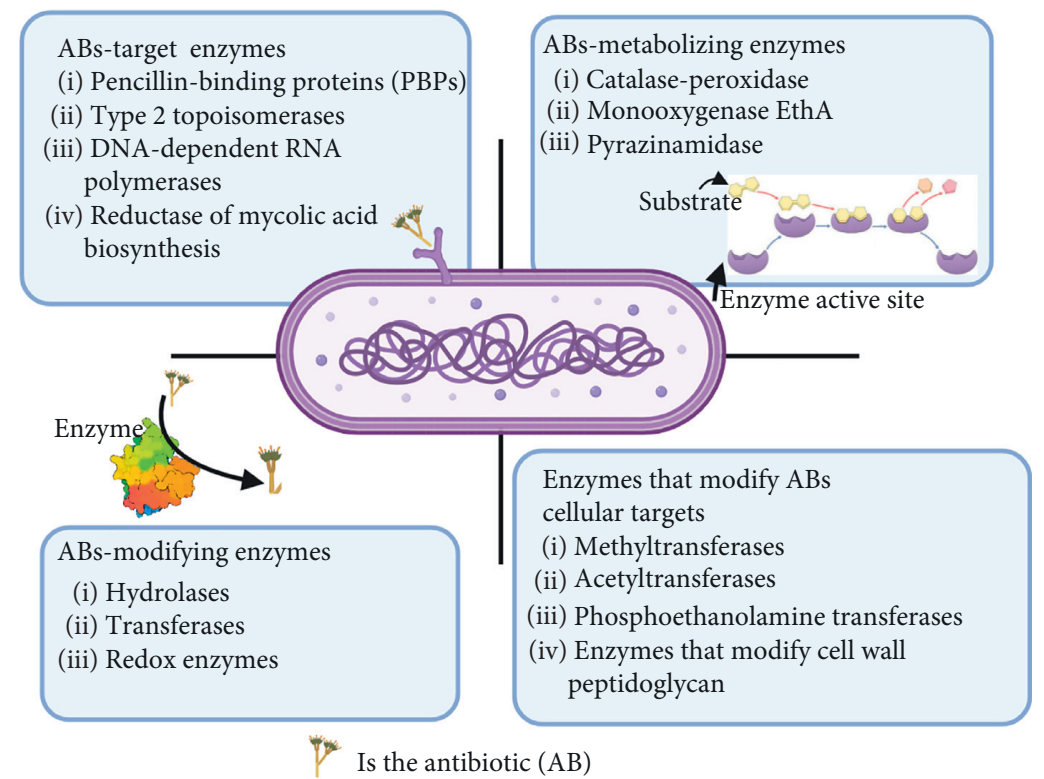

FIGURE 3: Bacterial enzymes that are involved in various mechanisms of microbial resistance.

enzymes as part of their mechanism. Thus, bacteria might resist antibiotics through structural changes or modification of the structural elements affected by antibiotics: for example, modification of ribosomes by methyltransferases [49].

Both Gram-positive and Gram-negative bacteria release enzymes to hydrolyze or modify antibiotic molecules, rendering them inactive [25]. Most antibiotics inactivated by this mechanism act through inhibition of bacterial protein synthesis [50]. Three types of enzymes are known to cause antibiotic resistance [51]:

(1) $\beta$-Lactamase enzymes hydrolyze the lactam ring in penicillin, cephalosporines, and carbapenems [52].
About 300 types of $\beta$-lactamases are identified based on structural and functional properties.

(2) Chloramphenicol acetyltransferase acetylates the hydroxyl group in chloramphenicol, making it unable to bind correctly to the target site. Haemophilus influenza, Gram-negative Bacilli, Enterobacteriaceae, Acinetobacter, and $P$. aeruginosa, resist chloramphenicol by using this mechanism [53].

(3) Aminoglycoside-modifying enzyme, S. aureus, S. pneumoniae, and E. faecalis fight antibiotics by producing enzymes that modify drug molecule and decrease its affinity for binding to $30 \mathrm{~S}$ subunits [54]. 
2.4. Alteration of the Binding Site. Bacteria may resist antibiotics by remodeling the bacterial target site in a way that decreases the binding affinity of antimicrobial agents [25]. Erythromycin binding interaction is markedly reduced by methylation of the target site, a peptidyl transferase enzyme [55]. Besides, Staphylococci acquired resistance to methicillin and oxacillin through mutational changes in penicillinbinding proteins (PBPs) [56]. Vancomycin-resistant Enterococci (VRE) counter antibiotic action by changing the amide linkage of the targeted enzyme into ester linkage, resulting in 1000 times less antibiotic binding affinity [57].

Helicobacter pylorus is a Gram-negative bacterium responsible for gastric infections such as gastritis, gastric ulcers, and gastric cancer [58]. H. pylori resist the first-line treatment agent "clarithromycin" via different mutations in the domain V of the 23S rRNA gene like A2142G, A2142C, or A2143G. These mutations decrease the affinity of bacteria to the antimicrobial agents [46]. Gram-positive bacteria alter antibiotic-binding sites making them ineffective, like modifying ribosomal targets to acquire resistance to macrolides, lincosamides, streptogramins, tetracyclines, and aminoglycosides [59].

Figure 4, adapted from Giannamaria Annunziato, represents a schematic illustration of antibiotic resistance mechanisms. Table 1 shows the mechanism of resistance and the type of antibiotics affected by common multidrug-resistant bacteria.

\section{Plant-Derived Compounds}

Since ancient times, herbal medicine was considered a cornerstone for treating various medical conditions such as cold, diarrhea, dental disease, and labor pain [66]. They represent patients' first treatment choice as they are associated with fewer side effects, and are cheap and affordable, especially in underdeveloped countries [67]. Even though approximately 17,000 plant species have been identified, only 3,000 species are in medical use [68]. However, according to the World Health Organization, $80 \%$ of people rely on folk medicine for their healthcare demands [66]. As a result, the search for medicinal plants with promising activity gains additional value. Plants are extensively investigated as a leading source for the synthesis of new therapeutic agents, and the possibility of using crude extracts in the treatment of different diseases [69].

Plants produce two types of metabolites: primary, which is essential for plant survival, and secondary, which resulted in response to plant interaction with the environment [70]. Primary metabolites are products of glycolysis, shikimate pathway, and tricarboxylic acid cycle, which aid in nutrition and reproduction [71]. In addition, they act as a precursor for thousands of secondary metabolites that are produced at different steps in primary metabolic pathways [72], which provokes the role of enzymatic activities against primary metabolites in the production of new compounds that support plant adaptation against biotic (e.g., bacteria, fungi, insect, and disease) and abiotic (e.g., injury, temperature, and moisture) stress [70].
Plant-derived compounds are secondary products of plant metabolism present in different parts of the plant, such as root, leaves, bark, flowers, seeds, and characterized by wide structural diversity $[73,74]$. For decades, plant-derived compounds acquired extra attention in the drug industry to enhance existing antibiotics' biological activity or as a potential source for new antimicrobial agents active against several pathogens, including $\operatorname{MDR}$ bacteria $(13,25,71)$. Based on their biosynthetic origin, plant phytochemicals can be divided into terpenoids, polyketides, phenylpropanoids, and alkaloids [71]. This review demonstrates the update in plant phytochemicals' antimicrobial activity along with their antibacterial mechanism of action against various MDR bacteria. In this review, plant-derived compounds were sectioned into alkaloids, polyphenols, terpenoids, sulfurcontaining compounds, and coumarins according to their chemical structures. Table 2 lists plants that reported antimicrobial activity against MDR bacteria from 2015 to 2021 .

3.1. Alkaloids. Alkaloids are organic nitrogenous compounds with substantial structural diversity [25]. The name "alkaloids" was introduced in 1819 by German chemist Carl Friedrich Wilhelm Meissner to refer to natural products of plant origin that showed basic properties similar to alkalis, but Friedrich Wilhelm Adam Sertürner is best known for his discovery of morphine in 1804; Sertürner isolated the first alkaloids [99]. These molecules' biosynthetic origin involves various organisms like bacteria, fungi, plants, and animals [100].

According to their biological sources, alkaloids may be classified into natural, semi-synthetic, and synthetic alkaloids, or based on their chemical structure into typical alkaloids with heterocyclic ring, and atypical alkaloid nonheterocyclic. This classification is further split into several classes: tropanes, indole, purines, imidazole, pyrrolidine, pyrrolizidine, isoquinoline, piperidine, and quinolizidine [100]. More than 18,000 types of alkaloids were identified from different sources [101].

The antimicrobial activity of alkaloids has been documented since the 1940s [102]. It has believed that the nitrogen atom is responsible for the bioactivity of this class [103]. Moreover, the antibacterial mechanism of alkaloidal phytochemicals against various infectious diseases is through efflux pump inhibition [25]. Table 3 shows the chemical structures of selected alkaloidal compounds with antimicrobial activity against MDR bacteria. For instance, sanguinarine (1) is an isoquinoline alkaloid present in various plants like Sanguinaria canadensis L., Fumaria officinalis L., Bocconia frutescens L., Chelidonium majus L., and Macleaya cordata (Willd.) R. Br. [115]. This compound exhibits broad pharmacological activity.

Concerning antimicrobial activity, sanguinarine isolated from root and aerial parts of Chelidonium majus L. exhibited a significant effect toward S. aureus strains with MIC $1.9 \mathrm{mg} /$ L. It is suggested that iminium bond, methoxy substitution, and the charge on quaternary nitrogen atom are responsible for this antibacterial action [104]. In a study, sanguinarine prevents bacterial cell division in $E$. coli by inhibiting 


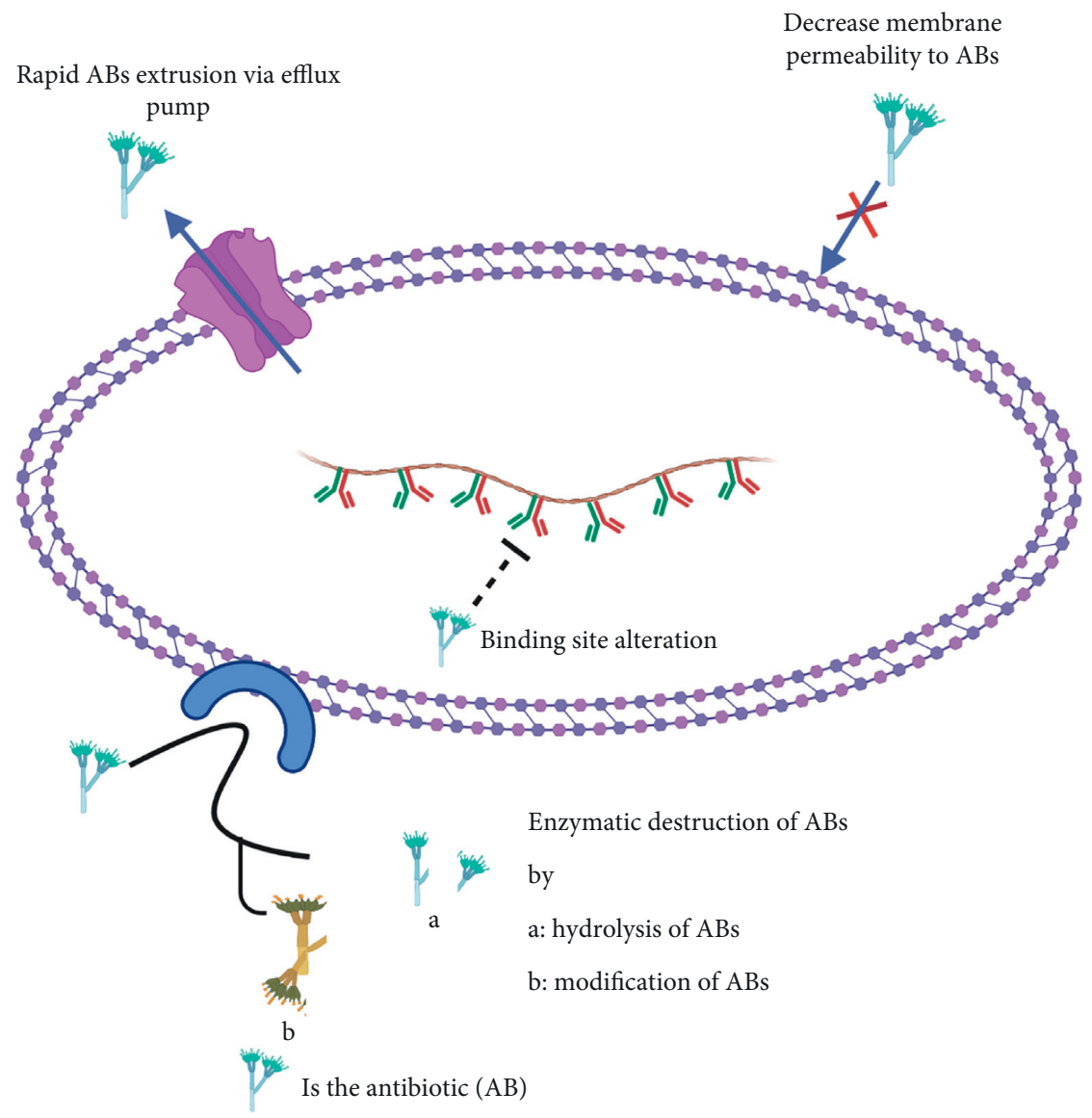

FIGURE 4: Schematic representation of antibiotic resistance.

cytokinetic $\mathrm{Z}$ ring formation [105]. This alkaloid effectively suppresses MRSA growth by weakening the bacterial cytoplasmic membrane, according to another study. When $M R S A$ is exposed to sanguinarine, it will produce autolytic enzymes to induce cell lysis [106]. In a recent study, sanguinarine exhibited potent inhibitory effects (MIC $7.8 \mu \mathrm{g}$ / $\mathrm{mL}$ ) on Providenica rettgeri-resistant isolates [116]. According to confocal laser scanning microscopy (CLSM), field emission scanning electron microscopy (FESEM), and crystal violet staining results, sanguinarine affected bacterial biofilm formation and resulted in a decrease in the intracellular ATP concentration [116].

The synergistic activity of sanguinarine was also documented. A study on the effect of sanguinarine, EDTA, and vancomycin on 34 strains of Gram-positive and Gramnegative bacteria was conducted. In the previous study, sanguinarine alone showed good activity against all strains with MIC $0.6-128 \mu \mathrm{g} / \mathrm{mL}$. Time kill study revealed that this alkaloid's bactericidal effect appeared after 4-6h. EDTA had a bacteriostatic effect, and the majority of Gram-negative bacteria resist vancomycin. However, this combination increases the sensitivity of resistant bacteria to vancomycin [117].

Similarly, a combination with streptomycin was studied. MIC and time-kill assay showed that sanguinarine, EDTA, and streptomycin had significant activity against
K. pneumoniae and E. coli but were inactive against MSRA and $S$. aureus [118]. These combinations proposed a possible strategy to overcome resistance. Another isoquinoline alkaloid is berberine [2], which is present in roots, stem-bark, and rhizome of various herbs such as Hydrastis canadensis L., Berberis aristata DC., Coptis chinensis Franch., Coptis japonica (Thunb.) Makino., and Phellodendron amurense Rupr. [119]. It has been known for a thousand years in China for its antimicrobial activity [119]. Interaction with bacterial DNA, inhibition of bacterial protein FtsZ responsible for cell division, and enzyme targeting are possible mechanisms for this antibacterial action [25].

Besides, berberine has a synergistic effect by enhancing the bacterial inhibition of some antibiotics [107]. In a previous study, a combination of berberine with ten different antibiotics was assessed for its antimicrobial activity against 14 Staphylococcus isolates. MIC values ranged from $16-512 \mu \mathrm{g} / \mathrm{mL}$. A $5 \mathrm{~mm}$ increase in the inhibition zone was observed with seven isolates, and berberine's combination with linezolid, cefoxitin, and erythromycin exhibited profound improvement in the antibacterial activity [107]. However, the mechanism underlying such effect is poorly understood.

However, in a recent study, it was noticed that berberine affects membrane integrity by altering the fatty acids contents in both saturated and unsaturated fatty acids, and 
TABLE 1: Mechanism of action and antibiotics affected by common MDR bacteria.

\begin{tabular}{|c|c|c|c|c|}
\hline MDR type & $\begin{array}{l}\text { WHO priority } \\
\text { category }\end{array}$ & Mechanism of resistance & ABs class & Reference \\
\hline $\begin{array}{l}\text { Pseudomonas } \\
\text { aeruginosa }\end{array}$ & Critical & $\begin{array}{l}\text { Efflux pump (MexAB-OprM) } \\
\text { Enzyme inactivation ( } \beta \text {-lactamase) } \\
\text { Alteration of membrane permeability }\end{array}$ & $\beta$-Lactams and penem groups of $\mathrm{ABs}$ & {$[60]$} \\
\hline $\begin{array}{l}\text { Acinetobacter } \\
\text { baumannii }\end{array}$ & Critical & $\begin{array}{c}\text { Enzyme inactivation (aminoglycoside- } \\
\text { modifying enzyme) } \\
\text { Efflux pump } \\
\text { Change membrane permeability }\end{array}$ & $\beta$-LactamsAminoglycosides & {$[61]$} \\
\hline $\begin{array}{l}\text { Klebsiella } \\
\text { pneumoniae }\end{array}$ & Medium & $\begin{array}{c}\text { Alteration of target site } \\
\text { Enzyme inactivation ( } \beta \text {-lactamase) } \\
\text { Efflux pump } \\
\text { Alteration of membrane permeability }\end{array}$ & $\begin{array}{c}3^{\text {rd }} \text { generation cephalosporins } \\
\beta \text {-Lactams Carbapenem }\end{array}$ & {$[62]$} \\
\hline $\begin{array}{l}\text { MRSA, VRSA, } \\
\text { VISA }\end{array}$ & High & $\begin{array}{l}\text { Binding site alteration Mutation in genes } \\
\text { involved in cell wall synthesisEfflux pump } \\
\text { (NorA) }\end{array}$ & $\begin{array}{c}\text { Methicillin } \\
\text { Oxacillin } \\
\text { Vancomycin } \\
\text { Penicillin } \\
\text { Cephalosporins } \\
\text { Carbapenem }\end{array}$ & {$[7]$} \\
\hline$V R E$ & High & Alteration of target site & $\begin{array}{l}\text { Most ABs } \\
\text { Vancomycin } \\
\text { Daptomycin } \\
\text { Linezolid } \\
\end{array}$ & {$[63]$} \\
\hline$C R E$ & Critical & $\begin{array}{l}\text { Enzyme inactivation }(\beta \text {-lactamase and } \\
\text { carbapenemase })\end{array}$ & $\begin{array}{c}\beta \text {-Lactams } \\
\text { Most ABs } \\
\text { Carbapenem } \\
\end{array}$ & {$[64]$} \\
\hline Escherichia coli & Critical & Efflux pump (AcrAB-TolC) & $\begin{array}{l}\text { Trimethoprim, amoxicillin, } \\
\text { gentamycin, tetracycline }\end{array}$ & {$[65]$} \\
\hline Helicobacter pylori & High & $\begin{array}{l}\text { Mutation in the domain } \mathrm{V} \text { of } 23 \mathrm{~S} \text { rRNA gene of } \\
\text { the bacteria }\end{array}$ & Clarithromycin & {$[46]$} \\
\hline
\end{tabular}

MRSA: methicillin-resistant staphylococcus aureus, VRSA: vancomycin-resistant staphylococcus aureus, VISA: vancomycin intermediate staphylococcus aureus, VRE: vancomycin-resistant enterococci, and CRE: carbapenem-resistant Enterobacteriaceae.

disrupting MRSA cell surface in a dose-dependent manner [120]. $\mathrm{K}^{+}$leakage and periplasmic alkaline phosphatase concentration had been assessed as an indication of membrane permeability changes. Furthermore, morphological alterations were detected in scanning electron microscopy (SEM) and transmission electron microscopy (TEM). Berberine-treated cells showed a nonspherical shape, and some of them have a doughnut-shaped structure without a central hole. In a recent report, berberine displayed a bacteriostatic effect against MRSA [108]. Molecular dynamics simulation data indicated that berberine could bind to the phenyl ring of Phe19 in PSM $\alpha 2$ via hydrophobic interaction. In addition, it can inhibit MRSA biofilm formation by influencing PSMs' aggregation into amyloid fibrils, which explains berberine's synergistic effect [108].

Solasodine (3) and Tomatidine (4) are essential glycoalkaloid metabolites isolated from the Solanaceae genus [121]. These molecules are well-known for decades for their biological activity and as starting compounds for synthesizing steroid drugs [122]. According to a recent study, genomic analysis was performed to identify the cause of resistance in MRSA. It was shown that modification of genetic sequences in bacterial ATP synthase confronted resistance to antibiotics [109]. Tomatidine appears to target this enzyme; thus, it could be considered a lead source to develop new antibiotics to combat bacterial resistance [109]. Tomatidine synergy was also evaluated against S. aureus, E. faecalis, P. aeruginosa, and E. coli using checkboard method and FIC indices. The results indicated that tomatidine has no antibacterial activity toward previously mentioned strains, but it inhibited $S$. aureus and $P$. aeruginosa growth combined with gentamycin or cefepime. A similar effect was recognized when $P$. aeruginosa and E. faecalis were exposed to tomatidine with ciprofloxacin or ampicillin [110].

Conessine (5) is another steroidal alkaloid isolated from the stem-bark of different species belonging to the Apocynaceae family, such as Holarrhena antidysenterica (Roth) Wall. ex A.DC. [111]. This plant's extract has been used in Thai folk medicine as antidiarrheal, antibacterial, and astringent. These effects can be attributed to steroidal alkaloid contents, mainly conessine [123]. Available studies indicated overexpression of efflux pumps, especially Mex-oprM confer $P$. aeruginosa resistance to Antibiotics [124, 125]. Thus, using EPI and existing antibiotics represent one of the strategies to restore antibiotic activity [126]. Coadministration of conessine with levofloxacin was tested against $P$. aeruginosa clinical isolates with a mutation in MexABOprM, MexcD-OprJ, and MeXEF-OprN efflux pumps [111]. MIC and time-kill study evaluated the efficacy of using 
TABLe 2: Plants reported with antimicrobial activity against MDR bacteria from 2015to 2021.

\begin{tabular}{|c|c|c|c|c|c|c|}
\hline Plant & Part & Active compound & $\begin{array}{c}\text { Mechanism of } \\
\text { action }\end{array}$ & Active against* & Findings & Reference \\
\hline Alkanna tinctoria & Leaves & $\begin{array}{c}\text { Alkaloids } \\
\text { Flavonoids } \\
\text { Carbohydrates }\end{array}$ & & $\begin{array}{l}\text { MRSA, E. coli, } \\
\text { P. aeruginosa, } \\
\text { A. baumannii }\end{array}$ & $\begin{array}{l}\text { MIC well diffusion } \\
\text { methodMBC model }\end{array}$ & [73] \\
\hline $\begin{array}{l}\text { Rhazya stricta } \\
\text { Decne. }\end{array}$ & Leaves & Alkaloids & $\begin{array}{c}\text { Cell } \\
\text { membrane } \\
\text { disruption }\end{array}$ & $\begin{array}{l}\text { MRSA, E. coli, } \\
\text { K. pneumoniae, VRE }\end{array}$ & TEM analysis & {$[74]$} \\
\hline $\begin{array}{l}\text { Holarrhena } \\
\text { antidysenterica }\end{array}$ & & Conessine alkaloid & $\begin{array}{l}\text { Efflux pump } \\
\text { inhibition }\end{array}$ & P. aeruginosa & $\begin{array}{c}\text { MIC model } \\
\text { NPN uptake assay } \\
\text { Active against RND } \\
\text { family }\end{array}$ & {$[75]$} \\
\hline $\begin{array}{l}\text { Allium sativum } \\
\text { L. (garlic) }\end{array}$ & Fruit & $\begin{array}{c}\text { Allicin (sulfur- } \\
\text { containing compound) }\end{array}$ & & P. aeroginosa & $\begin{array}{l}\text { MIC and MBC models } \\
\text { In vivo }\end{array}$ & {$[76]$} \\
\hline Oxalis corniculata & Leaves & & & $\begin{array}{c}\text { MDR Salmonella typhi } \\
\text { K. pneumoniae }\end{array}$ & $\begin{array}{c}\text { MIC and MBC well } \\
\text { diffusion methods }\end{array}$ & {$[18]$} \\
\hline Coula edulis Baill. & Fruit & $\begin{array}{l}\text { Alkaloids, flavonoids, } \\
\text { saponin } \\
\text { Cardiac glycosides }\end{array}$ & $\begin{array}{l}\text { Efflux pump } \\
\text { inhibition }\end{array}$ & $\begin{array}{c}\text { E. coli } \\
\text { K. pneumoniae }\end{array}$ & MIC and MBC models & {$[77]$} \\
\hline $\begin{array}{l}\text { Mangifera indica } \\
\text { L. }\end{array}$ & Bark & $\begin{array}{l}\text { Carotenoid } \\
\text { Tannin } \\
\text { Catechin } \\
\text { Polyphenol }\end{array}$ & $\begin{array}{l}\text { Efflux pump } \\
\text { inhibition }\end{array}$ & P. aeruginosa & MIC and MBC models & {$[77]$} \\
\hline Citrus sinensis & Peel & $\begin{array}{c}\text { Polyphenol } \\
\text { Catechin } \\
\text { Carbohydrates } \\
\end{array}$ & $\begin{array}{l}\text { Efflux pump } \\
\text { inhibition }\end{array}$ & E. coli & MIC and MBC models & {$[77]$} \\
\hline $\begin{array}{l}\text { Moringa oleifera } \\
\text { Lam. }\end{array}$ & Leaves & $\begin{array}{c}\text { Alkaloids, } \\
\text { Polyphenols } \\
\text { Flavonoids } \\
\text { Anthraquinones } \\
\text { Coumarin } \\
\text { Tannin, saponin } \\
\text { Terpenes, sterols } \\
\end{array}$ & & $\begin{array}{c}\text { P. aeruginosaKlebsiella } \\
\text { spp.E. coli }\end{array}$ & MIC and MBC models & {$[78]$} \\
\hline $\begin{array}{l}\text { Matricaria } \\
\text { recutita L. }\end{array}$ & Flowers & & & $\begin{array}{l}\text { P. aeruginosa } \\
\text { Klebsiella spp. } \\
\quad \text { E. coli }\end{array}$ & MIC and MBC model & {$[78]$} \\
\hline $\begin{array}{l}\text { Eleutherine } \\
\text { bulbosa (Mill.) } \\
\text { Urb. }\end{array}$ & $\begin{array}{c}\text { Bulb } \\
\text { extract }\end{array}$ & & & S. aureusShigella boydii & $\begin{array}{c}\text { MIC and MBC model } \\
\text { Time kill study } \\
\text { TLC-bioautography }\end{array}$ & {$[79]$} \\
\hline $\begin{array}{l}\text { Zanthoxylum } \\
\text { alatum }\end{array}$ & $\begin{array}{c}\text { Leaves, } \\
\text { stem }\end{array}$ & Fenchol, linalool & & E. coliK. pneumoniae & $\begin{array}{c}\text { In vitro model } \\
\text { MIC } \\
\end{array}$ & {$[80]$} \\
\hline $\begin{array}{l}\text { Cinnamomum } \\
\text { tamala }\end{array}$ & Leaves & Cinnamaldehyde & & MDR-H. pylori & In vitro model & {$[80]$} \\
\hline $\begin{array}{l}\text { Ocimum sanctum } \\
\mathrm{L} .\end{array}$ & Leaves & & & S. aureus-resistant strains & $\begin{array}{c}\text { In vitro model } \\
\text { MIC }\end{array}$ & {$[80]$} \\
\hline $\begin{array}{l}\text { Zanthoxylum } \\
\text { armatum DC. }\end{array}$ & Fruit & & & $\begin{array}{l}\text { E. faecium } \\
\text { S. aureus } \\
\text { K. pneumoniae } \\
\text { A. baumannii } \\
\text { P. aeruginosa }\end{array}$ & $\begin{array}{c}\text { MIC } \\
\text { Biofilm and quorum } \\
\text { sensitivity assay } \\
\delta \text {-Toxin inhibition } \\
\text { Mammalian } \\
\text { cytotoxicity study }\end{array}$ & {$[81]$} \\
\hline
\end{tabular}


TABle 2: Continued.

\begin{tabular}{|c|c|c|c|c|c|c|}
\hline Plant & Part & Active compound & $\begin{array}{l}\text { Mechanism of } \\
\text { action }\end{array}$ & Active against* & Findings & Reference \\
\hline $\begin{array}{l}\text { Adiantum } \\
\text { capillus-veneris L. }\end{array}$ & $\begin{array}{l}\text { Whole } \\
\text { plant }\end{array}$ & & & $\begin{array}{l}\text { E. faecium } \\
\text { S. aureus } \\
\text { K. pneumoniae } \\
\text { A. baumannii } \\
\text { P. aeruginosa } \\
\end{array}$ & $\begin{array}{c}\text { MIC } \\
\text { Biofilm and quorum } \\
\text { sensitivity assay } \\
\delta \text {-Toxin inhibition } \\
\text { Mammalian } \\
\text { cytotoxicity study }\end{array}$ & {$[82]$} \\
\hline $\begin{array}{l}\text { Artemisia } \\
\text { absinthium L. }\end{array}$ & $\begin{array}{l}\text { Aerial } \\
\text { parts }\end{array}$ & & & $\begin{array}{l}\text { E. faecium } \\
\text { S. aureus } \\
\text { K. pneumoniae } \\
\text { A. baumannii } \\
\text { P. aeruginosa }\end{array}$ & $\begin{array}{c}\text { MIC } \\
\text { Biofilm and quorum } \\
\text { sensitivity assay } \\
\delta \text {-Toxin inhibition } \\
\text { Mammalian } \\
\text { cytotoxicity study }\end{array}$ & {$[82]$} \\
\hline $\begin{array}{l}\text { Martynia annua } \\
\text { L. }\end{array}$ & Fruit & & & $\begin{array}{l}\text { E. faecium } \\
\text { S. aureus } \\
\text { K. pneumoniae } \\
\text { A. baumannii } \\
\text { P. aeruginosa }\end{array}$ & $\begin{array}{c}\text { MIC } \\
\text { Biofilm and quorum } \\
\text { sensitivity assay } \\
\delta \text {-Toxin inhibition } \\
\text { Mammalian } \\
\text { cytotoxicity study }\end{array}$ & {$[82]$} \\
\hline $\begin{array}{l}\text { Cynodon dactylon } \\
\text { (L.) Pers. }\end{array}$ & $\begin{array}{l}\text { Whole } \\
\text { plant }\end{array}$ & & & $\begin{array}{c}\text { MRSA } \\
\text { Imipenem-resistant } \\
P . \text { aeruginosa } \\
\text { MDR- salmonella typhi }\end{array}$ & MIC and MBC models & {$[82]$} \\
\hline $\begin{array}{l}\text { Ocimum } \\
\text { basilicum L. }\end{array}$ & & Phytol, cadinene & & $\begin{array}{l}\text { A. baumannii } \\
\text { E. coli }\end{array}$ & $\begin{array}{l}\text { MIC using broth } \\
\text { microdilution } \\
\text { technique }\end{array}$ & {$[83]$} \\
\hline $\begin{array}{l}\text { Plectranthus } \\
\text { barbatus } \\
\text { Andrews. }\end{array}$ & & $\begin{array}{l}\text { Phytol, camphor, } \\
\text { verbenone }\end{array}$ & & $\begin{array}{l}\text { A. baumannii } \\
\text { K. pneumoniae } \\
\text { E. coli } \\
\text { P. aeruginosa }\end{array}$ & $\begin{array}{l}\text { MIC using broth } \\
\text { microdilution } \\
\text { technique }\end{array}$ & {$[83]$} \\
\hline $\begin{array}{l}\text { Rosmarinus } \\
\text { officinalis L. }\end{array}$ & & $\begin{array}{l}\text { Phytol, camphor, } \\
\text { verbenone }\end{array}$ & & $\begin{array}{l}\text { A. baumannii } \\
\text { K. pneumoniae }\end{array}$ & $\begin{array}{l}\text { MIC using broth } \\
\text { microdilution } \\
\text { technique }\end{array}$ & {$[83]$} \\
\hline $\begin{array}{l}\text { Myrtus communis } \\
\text { L. }\end{array}$ & Seeds & $\begin{array}{c}\text { Gallic acid } \\
\text { Ellagic acid } \\
\text { Flavonoids } \\
\text { Fatty acid, tannin }\end{array}$ & & $\begin{array}{c}\text { S. aureus } \\
\text { P. aeruginosa } \\
\text { E. coli } \\
\text { S. enteric }\end{array}$ & MIC model & {$[60]$} \\
\hline $\begin{array}{l}\text { Cinnamomum } \\
\text { zeylanicum }\end{array}$ & Leaves & Polyphenol & & $\begin{array}{c}\text { S. aureus } \\
\text { P. aeruginosa } \\
\text { E. coli } \\
\text { S. enteric }\end{array}$ & MIC model & {$[60]$} \\
\hline $\begin{array}{l}\text { Syzygium legatii } \\
\text { Burtt Davy \& } \\
\text { Greenway. } \\
\text { Eugenia zeyheri } \\
\text { (Harv.) Harv. }\end{array}$ & Leaves & & & E. coli & $\begin{array}{l}\text { MICIn vitro toxicity } \\
\text { study using Caco-2 } \\
\text { cells }\end{array}$ & {$[84]$} \\
\hline $\begin{array}{l}\text { Peganum harmala } \\
\text { L. }\end{array}$ & Seeds & $\begin{array}{c}\text { Alkaloids } \\
\text { Harman, harmine } \\
\text { Harmaline, harmalol }\end{array}$ & & $M R S A$ & $\begin{array}{l}\text { MICMTT assay using } \\
\text { HEK-293 cells }\end{array}$ & {$[85]$} \\
\hline $\begin{array}{l}\text { Glycyrrhiza glabra } \\
\text { L. }\end{array}$ & $\begin{array}{c}\text { Fruit \& } \\
\text { leaves }\end{array}$ & $\begin{array}{l}\text { Alkaloids, saponin } \\
\text { Tannin, flavonoids } \\
\text { Phenols, coumarin, } \\
\text { terpenes }\end{array}$ & & P. aeruginosa & MIC model & {$[86]$} \\
\hline Ficus sycomorus L. & Leaves & $\begin{array}{l}\text { Flavonoids } \\
\text { Phenols }\end{array}$ & & $\begin{array}{c}\text { A. baumannii } \\
\text { Resistant } S \text {. aureus }\end{array}$ & MIC and MBC models & {$[87]$} \\
\hline
\end{tabular}


TABle 2: Continued.

\begin{tabular}{|c|c|c|c|c|c|c|}
\hline Plant & Part & Active compound & $\begin{array}{l}\text { Mechanism of } \\
\text { action }\end{array}$ & Active against* & Findings & Reference \\
\hline Syzigium cumini & Leaves & $\begin{array}{c}\text { Alkaloids } \\
\text { Flavonoids } \\
\text { Terpenoids } \\
\end{array}$ & & MRSAE. coli & MIC and MBC models & {$[88]$} \\
\hline $\begin{array}{l}\text { Punica granatum } \\
\text { L. }\end{array}$ & Peel & $\begin{array}{l}\text { Ellagic tannin } \\
\text { Ellagic acid } \\
\text { Gallic acid }\end{array}$ & & $P$. aeruginosa & MIC and MBC models & [89] \\
\hline $\begin{array}{l}\text { Camellia sinensis } \\
\text { (green tea) }\end{array}$ & Leaves & & & $M R S A$ & MIC model & {$[90]$} \\
\hline $\begin{array}{l}\text { Mentha longifolia } \\
\text { (L.) L. }\end{array}$ & $\begin{array}{l}\text { Arial } \\
\text { part }\end{array}$ & & & $V R E$ & MIC model & {$[90]$} \\
\hline $\begin{array}{l}\text { Croton } \\
\text { macrostachyus } \\
\text { hochst. ex Delile. }\end{array}$ & Leaves & $\begin{array}{c}\text { Triterpenes } \\
\text { Sterols, polyphenols } \\
\text { Saponins } \\
\end{array}$ & & MRSA & $\begin{array}{l}\text { MIC and MBCBroth } \\
\text { microdilution method }\end{array}$ & [91] \\
\hline $\begin{array}{l}\text { Catharanthus } \\
\text { roseus (L.) G.Don. }\end{array}$ & Leaves & $\begin{array}{c}\text { Alkaloids, triterpenes } \\
\text { Sterols, flavonoids } \\
\text { Polyphenols }\end{array}$ & & $M R S A$ & $\begin{array}{l}\text { MIC and MBCBroth } \\
\text { microdilution method }\end{array}$ & {$[91]$} \\
\hline $\begin{array}{l}\text { Paullinia pinnata } \\
\text { L. }\end{array}$ & Leaves & $\begin{array}{c}\text { Triterpenes } \\
\text { Sterols, polyphenols } \\
\text { Saponins }\end{array}$ & & $M R S A$ & $\begin{array}{l}\text { MIC and MBCBroth } \\
\text { microdilution method }\end{array}$ & [91] \\
\hline $\begin{array}{l}\text { Anacardium } \\
\text { occidentale L. }\end{array}$ & Leaves & $\begin{array}{l}\text { Alkaloids, saponin } \\
\text { Flavonoids, tannin } \\
\text { Phenol anthocyanin } \\
\end{array}$ & & E. coliK. pneumoniae & $\begin{array}{l}\text { MIC agar well diffusion } \\
\text { method }\end{array}$ & [92] \\
\hline $\begin{array}{l}\text { Thymbra spicata } \\
\text { L. }\end{array}$ & $\begin{array}{l}\text { Arial } \\
\text { parts }\end{array}$ & $\begin{array}{l}\text { Carvacrol, thymol } \\
\text { Camphor }\end{array}$ & & E. coli & $\begin{array}{c}\text { MIC and MBC } \\
\text { microdilution method }\end{array}$ & [93] \\
\hline $\begin{array}{l}\text { Lawsonia inermis } \\
\text { (henna) }\end{array}$ & Leaves & $\begin{array}{l}\text { Alkaloids, terpenoids, } \\
\text { phenolic compounds, } \\
\text { tannins, steroids, } \\
\text { anthraquinones }\end{array}$ & & $\begin{array}{c}\text { MRSA ATCC } 43300 \\
\text { K. pneumoniae } \\
\text { ATCC700603 } \\
\text { P. aeruginosa ATCC } 37853\end{array}$ & $\begin{array}{l}\text { MIC and MBC (agar } \\
\text { well diffusion and } \\
\text { colorimetric } \\
\text { microdilution } \\
\text { methods) }\end{array}$ & {$[94]$} \\
\hline $\begin{array}{l}\text { Azadirachta } \\
\text { indica (neem) }\end{array}$ & Leaves & $\begin{array}{l}\text { Alkaloids, terpenoids, } \\
\text { phenolic compounds, } \\
\text { tannins, steroids, } \\
\text { saponins, flavonoids }\end{array}$ & & $\begin{array}{c}\text { MRSA ATCC43300 } \\
\text { K. pneumoniae } \\
\text { ATCC700603 } \\
\text { P. aeruginosa ATCC } 37853\end{array}$ & $\begin{array}{l}\text { MIC and MBC (agar } \\
\text { well diffusion and } \\
\text { colorimetric } \\
\text { microdilution } \\
\text { methods) }\end{array}$ & {$[94]$} \\
\hline Piper betle Linn. & Leaves & & & P. aeruginosa TISTR1287 & $\begin{array}{l}\text { Agar-disc diffusion } \\
\text { method } \\
\text { Broth dilution assay } \\
\text { (MIC and MBC) }\end{array}$ & [95] \\
\hline Cistus salviifolius & & $\begin{array}{c}\text { Hydrolysable tannins, } \\
\text { flavonoids (myricetin } \\
\text { and quercetin) }\end{array}$ & & $M R S A$ & $\begin{array}{l}\text { Disc-dilution method, } \\
\text { microdilution method }\end{array}$ & [96] \\
\hline Punica granatum & & $\begin{array}{l}\text { Hydrolysable tannins } \\
\text { (punicalin and } \\
\text { punicalagin) }\end{array}$ & & MRSA & $\begin{array}{l}\text { Disc-dilution method, } \\
\text { microdilution method }\end{array}$ & {$[96]$} \\
\hline Platanus hybrida & Fruits & Phenolic compounds & & $\begin{array}{c}\text { E. faecium, E. faecalisP. } \\
\text { aeruginosa, K. pneumoniae }\end{array}$ & $\begin{array}{l}\text { Kirby-Bauer disc } \\
\text { diffusion method }\end{array}$ & [97] \\
\hline $\begin{array}{l}\text { Syzygium } \\
\text { aromaticum }\end{array}$ & $\begin{array}{l}\text { Flower } \\
\text { buds }\end{array}$ & & & K. pneumoniaeS. aureus & $\begin{array}{l}\text { Disc-diffusion method } \\
\text { (MIC and MBC), } \\
\text { scanning electron } \\
\text { microscopy, DNA } \\
\text { apoptosis }\end{array}$ & [98] \\
\hline Acacia nilotica & Seeds & & & S. aureus & $\begin{array}{l}\text { Disc-diffusion method } \\
\text { (MIC and MBC), } \\
\text { scanning electron } \\
\text { microscopy, DNA } \\
\text { apoptosis }\end{array}$ & [98] \\
\hline
\end{tabular}

${ }^{*}$ All bacteria strains are multidrug resistant. 
TABLE 3: Chemical structures of alkaloidal compounds with antimicrobial activity against MDR bacteria.<smiles></smiles>

[1]<smiles></smiles>

[2]

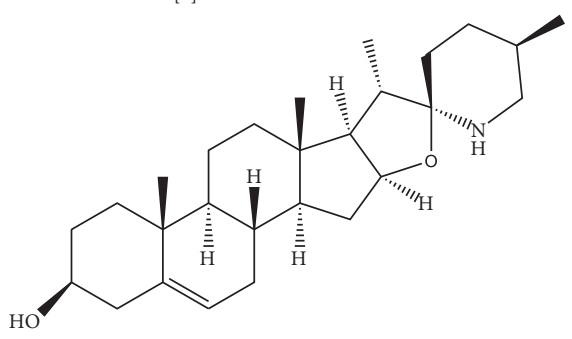

[3]

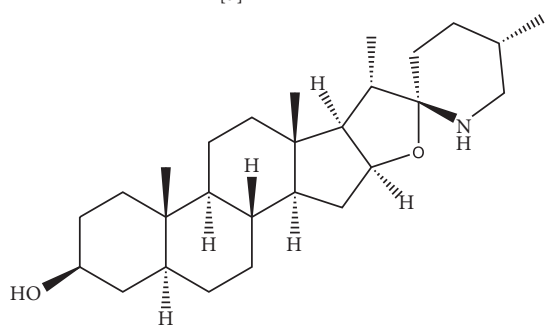

[4]

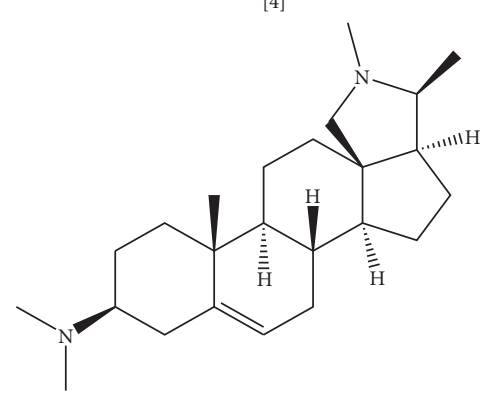

[5]

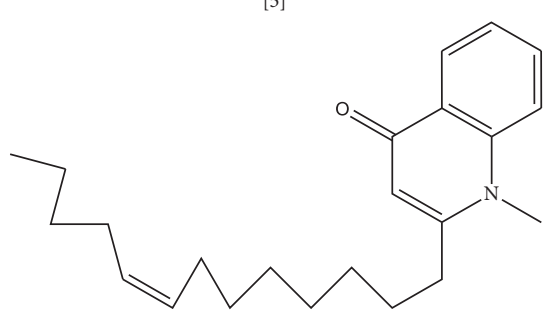

[6]
MRSA, E. coli, S. aureus

[104-106]

E. coli, MRSA, synergistic action against Staphylococcus spp.

$[25,107,108]$

MRSA

[109]

S. aureus, E. faeclis, P. aeruginosa, E. coli

[110]

P. aeruginosaA. baumannii

[111, 112]

M. tuberculosis 
TABLE 3: Continued.

Chemical structure Active against

levofloxacin alone and with alkaloid combination. The result showed that conessine successfully retrieved levofloxacin efficacy against $P$. aeruginosa strain with efflux pumps mediated resistance [111]. In an analogous manner, conessine has a synergistic effect when combined with novobiocin or rifampicin in treating extensively drug-resistant A. baumannii infection [112].

Anti-malarial compounds such as quinoline alkaloid evocarpine (6) and quinolines like dictamnine and masculine are known to have noticeable antimicrobial activity $[113,127]$. Evocarpine exhibited higher antibacterial activity than isoniazid in the treatment of M. tuberculosis infection. This effect relates to the inhibition of enzymes required for bacterial cell wall synthesis [113]. Coadministration of ergot alkaloid chanoclavine (7) with tetracycline markedly suppresses resistant E. coli growth [114]. Although chanoclavine has no antibacterial activity alone, with tetracycline it shows considerable synergy. The mechanism underlying this action is due to chanoclavine-mediated inhibition of bacterial efflux pump, which is ATPase dependent. In silico docking showed the ability of chanoclavine to bind with various proteins involved in drug resistance [114]. Alkaloidal antibacterial activity of two plant leaves extracts (Callistemon citrinus (Curtis) Skeels. and Vernonia adoensis) were tested against MDR $S$. aureus and $P$. aeruginosa. Callistemon citrinus (Curtis) Skeels. showed potent antibacterial activity with $\mathrm{MIC}=0.0025 \mathrm{mg} / \mathrm{mL}$, and efflux pump inhibition caused by rhodamine $6 \mathrm{G}$ accumulation of $121 \%$ compared to control [128].

Tetrahydrosecamine (piperidine alkaloid) and Strictanol (indole alkaloid) isolated from the leaves of Rhazya stricta Decne. exhibited significant antimicrobial activity toward MRSA, E. coli, and P. aeruginosa through disruption of the bacterial cell membrane [74]. Similarly, two isoquinoline alkaloids isolated for the first time from Zanthoxulum tingoassuiba root bark, dihydrocheleryhtrine, and N-methylcanadine showed good antibacterial activity (MIC $60 \mu \mathrm{g} / \mathrm{mL}$ ) against MRSA [129]. Four papaver species from the Papaveraceae family grown naturally in Iran were tested for the antimicrobial activity for both Gram-positive and Gramnegative pathogens. Benzylisoquinoline alkaloids were identified from Papaver macrostomum Boiss. \& A.Huet., Roemeria refracta DC., Papaver somniferum L., and Glaucium grandiflorum tissues. The alkaloidal extracts of these plants were tested against $P$. aeruginosa, $S$. aureus, and
K. pneumoniae. All extracts displayed considerable inhibitory effects for Gram-positive pathogens more than Gramnegative bacteria. Roemeria refracta DC. alkaloid has a stronger inhibitory effect toward $S$. aureus than other species (MIC $0.065 \mu \mathrm{g} / \mathrm{mL}$ ) [130].

In the Middle East, Salvadora persica is traditionally used as a chewable stick to ensure oral hygiene. Methanolic and aqueous extract of this plant were studied for their antimicrobial activity against MDR $S$. aureus and $P$. aeruginosa. Alkaloids, saponin, flavonoids, terpenoids, and volatile oils were isolated from S. persica extract [131]. The aqueous extract showed better inhibition with MIC equal to $2.49 \mathrm{mg} /$ $\mathrm{mL}$ for $S$. aureus and $7.34 \mathrm{mg} / \mathrm{mL}$ for $P$. aeruginosa. However, the specific compound responsible for this activity was not identified yet [60].

Black zira essential oils exhibited bacteriostatic and bactericidal activity toward several pathogens, including $P$. aeruginosa and E. coli, with MIC ranging from $1 \mathrm{mg} / \mathrm{mL}$ to $8 \mathrm{mg} / \mathrm{mL}$ and MBC values ranging between 1 and $16 \mathrm{mg} / \mathrm{mL}$. Phytochemical analysis revealed the presence of alkaloids, saponin, flavonoids, tannin, and phenols [131]. The antimicrobial activity of leaves' extracts of Carica papaya L., Datura stramonium L., and fruit extract of Piper nigrum L. was tested against Gram-positive and Gram-negative bacteria including MDR $S$. aureus, $P$. aeruginosa, A. baumannii, and E. coli. All extracts showed better activity against Gram-positive bacteria. However, Piper nigrum extract had no activity toward MDR. Ethanolic extracts of Carica papaya L. and Datura stramonium L. displayed larger zone of inhibition than methanol extracts. The phytochemical screening for both plants indicated the presence of alkaloids, steroids, glycosides, phenols, saponin, and carotenoids with tannin present only in Datura extract [132]. Several alkaloids were identified in a southeast Asian plant, Piper sarmentosum Roxb.; some are present in roots and stems like langkamide, piplartine, and trimethoxycinnamic acid, while amide alkaloid compounds were isolated from plant leaves. Methanolic extracts of the leaves exhibited good antibacterial activity, including MDR pathogens such as MRSA and E. coli with MIC $100 \mathrm{mg} / \mathrm{mL}$ [133].

3.2. Polyphenols. Polyphenols are a large group of secondary plant metabolites with phenolic pharmacophore [134]. More than 8000 polyphenols were identified with biological 
activity mainly as antioxidants that protect plant and human cells from free radical damage [135]. They are classified into four categories according to the number of phenolic groups and the structural elements as follows: (1) flavonoid, which further split into flavones, isoflavones, flavonols, flavanones, chalcones, and catechins; (2) stilbenes with resveratrol as the main compound; (3) lignans and finally (4) phenolic acids like hydroxybenzoic acid and hydroxycinnamic acid [136].

Besides polyphenols' central role as an antioxidant, antiinflammatory, antiallergic, anticancer, antihypertensive, and antimicrobial activities were also documented [137]. Figure 5 illustrates the chemical structures of some polyphenols with documented antimicrobial activity against MDR. Although the exact mechanism for polyphenols' antimicrobial action is not fully understood, several studies suggested that at the cellular level, polyphenols bind to bacterial enzymes via a hydrogen bond, inducing several modifications in cell membrane permeability and cell wall integrity [138].

One of the most abundant flavonoids present in food are flavanols, which have potent antimicrobial activity against Gram-positive and Gram-negative pathogens, including resistant strains [139]. Examples of this group include quercetin (8) and kaempferol (9). In one study, a combination of quercetin with amoxicillin exhibited synergistic activity against $S$. epidermidis isolates that are resistant to amoxicillin alone; however, the activity was markedly reserved when quercetin was added [140]. According to the study, quercetin inhibited beta-lactamase and bacterial peptidoglycan synthesis, as well as increased cell membrane permeability and decreased fatty acids in bacterial cells [140]. Similarly, quercetin's synergy with levofloxacin, ceftriaxone, gentamycin, tobramycin, and amikacin has been tested against $P$. aeruginosa. The biofilm formation and biofilm cell viability had been markedly affected by this combination with $\geq 80 \%$ inhibition. Bactericidal effect was significant as well with (68 to $85 \%$ ) cell killing [141]. In another study, a combination of low-dose quercetin and tetracycline exhibited bactericidal action against antibiotic-resistant E. coli in a mouse-infected model. As a mechanism, quercetin disrupted the bacterial cell envelope, resulting in increased cell permeability and lysis [142]. These results encourage more focus on combining antibiotics with phytochemicals as a new approach to combat bacterial resistance.

Kaempferol and its derivatives isolated from Bryophyllum pinnatum displayed significant antibacterial activity against several pathogens, including antibiotic-resistant $S$. aureus and $P$. aeruginosa [143]. For the last decade, kaempferol acquired extra attention after its activity against MRSA had been proven [144]. This action's mechanism is thought to be through kaempferol-mediated inhibition of NorA efflux pump [145]. Om Prakash et al. studied the effect of quercetin and kaempferol combination with regular antibiotics in the antibacterial activity of these antibiotics against MRSA [146]. According to the study, antibiotics like cefixime, ceftriaxone, ciprofloxacin, cephradine, methicillin, ampicillin, and amoxicillin, when mixed with quercetin, kaempferol, or quercetin + kaempferol, revealed remarkable
MIC values against MRSA, which is usually resistant to these antibiotics when administered alone. However, a combination of classical antibiotics with these flavonoids restores their antibacterial activity [146].

Camellia sinensis (green tea) is a plant well-known for its antioxidant and antimicrobial properties against various pathogens, including resistant $S$. aureus and $P$. aeruginosa [147]. In a previous study, green tea extract was reported to be active against 11 isolates of resistant $E$. coli in patients who suffered from UTI infections in Nepal (MIC $600 \mu \mathrm{g} / \mathrm{mL}$ ) [148]. Catechin (10) is a polyphenol abundant in green tea and accountable for the plant's activity. Four forms of catechin are present in green tea; epicatechin (EC) (11), epigallocatechin (EGC) (12), EC-3-gallate (ECG) (13), and EGC-3-gallate (EGCG) (14) [147]. Several studies indicated the pharmacological importance of catechin phytochemical $[147,149,150]$. In a study, catechin showed metal-chelating properties making it more effective as an antioxidant than vitamin C [149]. Moreover, green tea extract was shown to be active against $P$. aeruginosa in several studies [147]. Catechin gallates effectively reverse MRSA resistance through NorA efflux pump inhibition. It has two different binding sites in the NorA substrate and causes EPI at high concentrations [151].

Chalcones are considered important secondary metabolites and precursors of flavonoids and isoflavonoids in plants [152] that produce their antimicrobial activities through NorA efflux pump inhibition [153]. Chalcone (15) and dihydrochalcone (16) isolated from the ethanolic root extract of Uvaria chamae P. Beauv. were assessed for the first time by Koudokpon et al. for their antibacterial activity against Gram-positive MDR pathogens [154]. Ten chalcones were identified by ion mobility MS analysis with significant inhibition of VRE, MRSA, and S. aureus growth [154]. Some research documented a considerable increase in antibacterial activity of chalcones in combination with antibiotics. An example is a chalcone isolated from traditional African and American herb roots, Sophora flavescens Aiton. [155]. Following the previously mentioned study, 7,9,2',4'-tetrahydroxy-8-isopentenyl5-methoxychalcone was found to be active against MRSA and VRE alone or in combination with ampicillin or gentamycin [155]. 4' $4^{\prime}, 6^{\prime}$-Dihydroxy-3', $5^{\prime}$-dimethyl-2' methoxychalcone from Dalea versicolor Zucc. was found to enhance erythromycin activity by reducing MIC from $0.4-0.1 \mu \mathrm{g} / \mathrm{mL}$ in another study [153].

Several studies reported that synthesized flavonoids' derivatives could be more effective as antibacterial agents than natural flavonoids [156-158]. Seventeen chalcone derivatives were synthesized and tested for their antimicrobial activity against eleven bacteria strains, including $S$. aureus and E. faecalis [158]. All compounds significantly inhibited bacterial growth, with one compound (dibromo derivative) (17) showing similar activity comparable to the nalidixic acid reference drug (MIC $25 \mu \mathrm{g} / \mathrm{mL}$ ). Quantitative structural activity relationship and docking study of these compounds indicated that binding to the active site of bacterial penicillin-binding protein PBP-1b gives antibacterial activity to these synthesized derivatives [158]. 
<smiles>O=c1c(O)c(-c2ccc(O)c(O)c2)oc2cc(O)cc(O)c12</smiles>

[8]<smiles>O=c1c(O)c(-c2ccc(O)cc2)oc2cc(O)cc(O)c12</smiles>

[9]<smiles>Oc1cc(O)c2c(c1)O[C@H](c1ccc(O)c(O)c1)[C@H](O)C2</smiles>

[10]<smiles>Oc1cc(O)c2c(c1)O[C@H](c1ccc(O)c(O)c1)[C@H](O)C2</smiles>

[11]<smiles>O=C(O[C@H]1Cc2c(O)cc(O)cc2O[C@@H]1c1ccc(O)c(O)c1)c1cc(O)c(O)c(O)c1</smiles>

[13]<smiles>Oc1cc(O)c2c(c1)O[C@H](c1cc(O)c(O)c(O)c1)[C@H](O)C2</smiles>

[12]<smiles>O=C(O[C@H]1Cc2c(O)cc(O)cc2O[C@@H]1c1cc(O)c(O)c(O)c1)c1cc(O)c(O)c(O)c1</smiles>

[14]<smiles>O=C(CCc1ccccc1)c1ccccc1</smiles>

[16]<smiles>COc1cc(C(Br)C(Br)C(=O)c2ccccc2)ccc1O</smiles>

[17]

(a)

FIgURE 5: Continued. 
<smiles>CC(C)=CCc1cc2c(=O)cc(-c3ccc(O)cc3)oc2cc1O</smiles>

[18]<smiles>CC(C)=CCc1c(-c2ccc(O)cc2O)oc2cc(OO)c(/C=C\C(C)C)c(O)c2c1=O</smiles>

[19]<smiles>O=c1cc(-c2ccccc2)oc2cc(O)c(O)c(O)c12</smiles>

[20]<smiles>Oc1ccc(/C=C/c2cc(O)cc(O)c2)cc1</smiles>

[21]

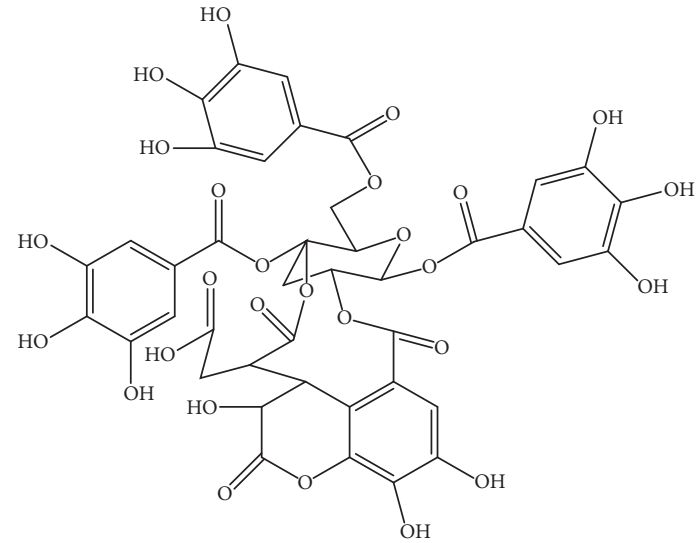

[22]<smiles>O=C(Oc1cc(C(=O)OC2O[C@H](COC(=O)c3ccc(OC(=O)c4cc(O)c(O)c(O)c4)c(O)c3O)[C@H](OC(=O)c3cc(O)c(O)c(O)c3)[C@@H](OC(=O)c3cc(O)c(O)c(OC(=O)c4cc(O)c(O)c(O)c4)c3)[C@H]2OC(=O)c2cc(O)c(O)c(OC(=O)c3cc(O)c(O)c(O)c3)c2)cc(O)c1O)c1cc(O)c(O)c(O)c1</smiles>

[23]<smiles>COc1cc(/C=C/C(=O)O)ccc1O</smiles>

$[25]$

(b)

FIGURE 5: Chemical structures of selected alkaloids with antimicrobial activity against MDR bacteria. 
Different mechanisms aim to explain the antibacterial action of flavone and isoflavone phytochemicals [139]. Flavone may form a complex with bacterial cell wall components preventing further adhesion. An example of this mechanism is the licoflavone (18) bioactive compound isolated from the flowers of the Retama raetam, which actively inhibits $E$. coli growth with MIC $7.81 \mu \mathrm{g} / \mathrm{mL}$ [159]. Inhibition of bacterial enzymes is another mechanism as in artocarpin (19) compound extracted from Artocarpus anisophyllus Miq. leaves. This phytochemical exerts an antibacterial effect against MDR E. coli and P. aeruginosa [160]. Several studies in the synergistic effect of flavones with wellknown antibiotics had been assessed, including the inhibition of bacterial efflux pumps, and increasing antibiotic susceptibility [139]. Baicalein (20) is a trihydroxyflavone present in the roots of Scutellaria baicalensis Georgi. and possesses various biological activities such as antiviral, antibacterial, anti-inflammatory, anticancer, and antioxidant properties [161]. Baicalein restores tetracycline and $\beta$-lactams activity against MRSA, and E. coli through the EPI mechanism [162]. In another study, the combination of baicalein with oxacillin and vancomycin were evaluated against MRSA and VRE. As a mechanism, this combination has a bactericidal effect via inhibition of bacterial cell wall synthesis. Time kill study revealed that the bacterial growth remarkably attenuated after 2-6h [142].

Resveratrol (21) is a stilbene polyphenol that has recently gained extra attention for its benefits [163]. It has been studied for its antimicrobial potential alone and in combination with classical antibiotics [163]. Resveratrol exhibits moderate antibacterial activity toward Gram-positive S. aureus and E. faecalis (MIC 100-200 $\mu \mathrm{g} / \mathrm{mL}$ ); however, it is less active against Gram-negative species such as $E$. coli and K. pneumoniae (MIC $>200 \mu \mathrm{g} / \mathrm{mL}$ ) [164]. As a mechanism, resveratrol inhibits biofilm production, and bacterial ATP synthase, thus decreasing the energy consumption required for bacterial growth. It also decreases bacterial toxin production, motility, and intervention with quorum sensing [163]. Eight bacterial strains, including MDR clinical isolates, were chosen to study resveratrol's in vitro antimicrobial activity [165]. According to the study, resveratrol displayed strong antimicrobial activity against $P$. aeruginosa and $S$. aureus (MIC $1.6 \mu \mathrm{g} / \mathrm{mL}$ and $1.7 \mu \mathrm{g} / \mathrm{mL}$ ), respectively, compared to the norfloxacin reference drug (MIC $4.8 \mu \mathrm{g}$ / $\mathrm{mL}$ ) [165]. In another study, resveratrol extracted from seeds of pinot noir grapes inhibited Campylobacter jejuni isolates' growth. Resveratrol acts as EPI for the CmeABC efflux pump responsible for the extrusion of antibiotics across the bacterial outer membrane [166].

Tannin is a water-soluble polyphenol commonly isolated from woody plants. It has been classified into two types: hydrolysable tannin, which is usually an ester of phenolic acid, and nonhydrolysable (condensed), a more abundant type of tannin usually derived from flavonoid dimers such as procyanidin [167]. Chebulinic acid (22) is hydrolysable tannin isolated from seeds of Euphoria longana, Terminalia chebula fruits, and leaves of Terminalia macroptera Guill. \& Perr. Kunal patel et al. studied the binding affinity of 179 compounds to Mycobacterium DNA gyrase enzyme including chebulinic acid [168] which displayed a significant inhibitory effect on quinolone-resistant DNA gyrase. However, this study is based on virtual screening; further in vitro study is required to confirm this activity [168]. In another study, chebulinic acid extracted from Terminalia chebula showed potent antimicrobial action toward MDR A. baumannii [169].

Tannic acid (23), another form of tannins, was proven to have efflux modulating properties against MRSA [170]. In a study, MRSA parent strains showed no efflux pump activity. However, with continuous use of fusidic acid, mutation emerges, and these mutant strains develop efflux pump type of resistance. It was shown that tannic acid administration with fusidic acid prevents mutation by acting as an alternative target for bacteria and promoting cell lysis [170]. Phenolic acids are natural compounds with one carboxylic acid functionality and many hydroxyl groups attached to the aromatic ring [171]. They could be derivatives of benzoic acid like gallic, protocatechuic, and $p$ hydroxybenzoic acids or cinnamic acids such as ferulic acid and $p$-coumaric acid [171]. Gallic acid (24) and ferulic acid (25) cause irreversible changes in E. coli and P. aeruginosa membranes through the reduction in negative surface charge and pore formation in cell membrane along with hydrophobicity changes, resulting in bacterial intracellular contents leakage [171].

A large-scale study was conducted to screen the antimicrobial effect of 239 traditional Chinese extracts against multidrug-resistant S. aureus. Some of these extracts reveal promising antibacterial activity (MIC ranging from 0.1 to $12.5 \mathrm{mg} / \mathrm{mL}$ and an $\mathrm{MBC}$ range of $0.78-25 \mathrm{mg} / \mathrm{mL}$ ) as well as low cytotoxicity with median lethal concentration $\left(\mathrm{LC}_{50}\right)>100 \mu \mathrm{g} / \mathrm{mL}$. The most active extracts include Rhus chinensis Mill., Ilex rotunda Thunb., Leontice kiangnanensis P.L.Chiu., Oroxylum indicum Vent., Isatis tinctorial L., Terminalia chebula Retz., Acacia catechu (L.f.) Willd., Spatholobus suberectus Dunn., Rabdosia rubescens (Hemsl.) H.Hara., Salvia miltiorrhiza Bunge., Fraxinus fallax Lingelsh., Coptis chinensis Franch., Agrimonia Pilosa Ledeb., and Phellodendron chinense C.K.Schneid. [172]. Further study is required to determine the active phytochemicals in these extracts and the potential mechanisms for their action.

3.3. Sulfur-Containing Compounds. Volatile compounds with organo-sulfur structure have been proven to have broad antibacterial activity against Gram-positive and Gramnegative pathogens [173]. Little is known about their mechanism of action; however, the disulfide bond's presence confers a great role in their effectiveness [173]. Examples of these compounds are allicin, isothiocyanate, cysteines, and ajoene (Figure 6 lists the chemical structure of selected sulfur-containing compounds reported with antimicrobial activity). Garlic (Allium sativum L.) is a plant well recognized among other Allium species for its antimicrobial activity towards resistant bacteria [174]. This activity is related to garlic's organo-sulfur constituents, mainly allicin (26), produced in response to garlic tissue damages and gives the crushed garlic its characteristic odor [174]. 


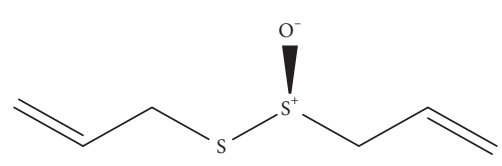

[26]

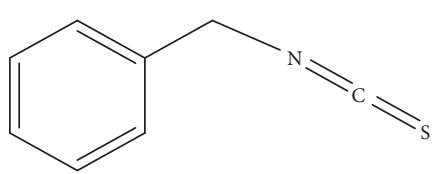

[29]

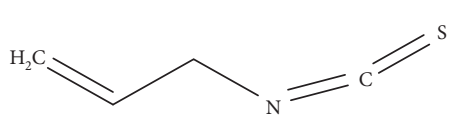

[27]

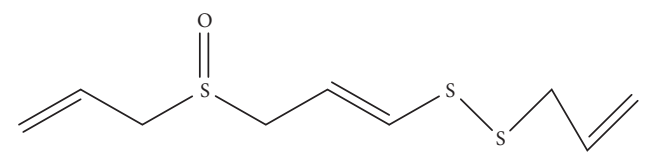

[30]

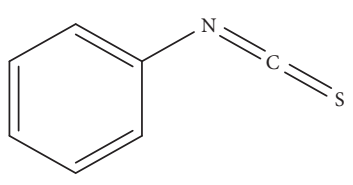

$[28]$

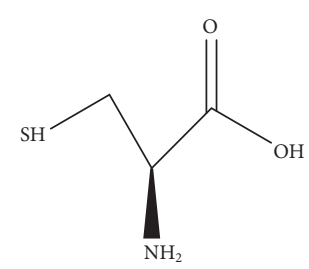

$[31]$

FIGURE 6: The chemical structures of selected sulfur-containing compounds reported with antimicrobial activity against MDR bacteria.

Several studies reported that allicin could inhibit MDR bacterial growth such as S. aureus, E. coli, MRSA, and $P$. aeruginosa; however, this effect is bacteriostatic, which suggested using allicin in combination with other antibiotics $[175,176]$. As a mechanism, allicin inhibits bacterial enzymes like alcohol dehydrogenase, RNA polymerase, DNA gyrase, as well as inhibition of biofilm formation, DNA, and protein synthesis $[175,177]$. Allicin presence in garlic was first reported by Cavallito and Bailey in 1944 [178]. It is a chemically unstable compound that rapidly decomposes to sulfide compounds such as ajoenes, diallyl sulfide (DAS), and diallyl disulfide (DADS) [175]. Thus, five analogues of allicin had been synthesized to overcome the stability problem [179]. The efficacy of these analogues was checked against bacteria and fungi, including E. coli and Pseudomonas spp. According to the study these analogues exhibited similar activity compared to allicin. The most potent antimicrobial activity was found in vapor form, concluding the use of allicin or its analogues in the gas phase as an alternative to antibiotics in the treatment of lung infection with MDR bacteria [179].

Plant family Brassicaceae represents several vegetables such as broccoli, mustard, cauliflowers, and cabbage. This family produces glucosinolates metabolites in response to tissue injury hydrolyzed by myrosinase enzyme into isothiocyanates (ITCs) [180]. Several natural as well as synthetic ITCs have been identified as potential antimicrobials against human pathogens, including resistant bacteria [181]. The antimicrobial activity of allyl isothiocyanate AITC (27) was isolated from a Japanese flower (Wasabia japonica (Miq.) Matsum.), and its synthetic analogue was evaluated against E. coli strain and S. aureus [182]. AITCs and their analogues sufficiently inhibit these bacteria's growth for up to $12 \mathrm{~h}$. [182]. However, according to another study, this phenotype's efficacy is 100 -fold less than polymyxin B antibiotic [183]. AITC affects bacterial membrane, similarly increasing cellular substance leakage to polymyxin B [183].

Another study on the AITC mechanism of action indicates that AITC may inhibit bacterial DNA synthesis by interfering with the sulfhydryl group in thioredoxin, promoting inferior enzyme activity, and interfering with the sulfhydryl group of acetate kinase, affecting energy metabolism of bacteria [184]. A previous study tested the effect of allyl isothiocyanate AITC, benzyl isothiocyanate BITC, and phenyl isothiocyanate PITC on $P$. aeruginosa [185]. These ITCS represent the major components of ITCs isolated from Tropaeolum majus L. and Armoracia ruticana plants. AITC, BITC, and PITC remarkably inhibited mature biofilm formation and showed synergy with meropenem by increasing its efficacy toward $P$. aeruginosa biofilm [185].

Phenyl isothiocyanate PITC (28) has proven to have moderate activity against S. aureus and E. coli (MIC $1000 \mu \mathrm{g} /$ $\mathrm{mL}$ ) [186]. It modulates bacterial membrane changes through the interaction of electrophilic compounds with bacterial cells leading to decreased negative charges at the bacterial surface and increased surface hydrophilicity and electron donation. These changes trigger cellular disruption and loss of membrane integrity resulting in cell death. In addition, the synergism of PITC with ciprofloxacin and erythromycin was tested. The previous study recommended using PITC in combination with one of these two antibiotics as it enhances the antimicrobial activity of these antibiotics toward resistant S. aureus and E. coli [186]. ITCs isolated from Cruciferous species were tested for their antimicrobial effect against 15 MRSA clinical isolates from diabetic foot ulcers [187]. The antibacterial activity of ITCs mainly depends on their structural properties, with benzyl isothiocyanate BITC being the most active (bacteriocidal effect is up to 87\%) [187].

As a mechanism, BITC structure has both lipophilic and electrophilic characteristics promoting disruption of bacterium ability to maintain membrane integrity through penetration of bacterial outer membrane [188]. Ajoene (30) is an organosulfur phenotype present in garlic extract and has two forms, E-ajoene and Z-ajoene [189]. This compound has broad pharmacological effects as an anticancer, antioxidant, antithrombosis, antiviral, antiparasitic and antimicrobial agent [190]. Although there is little knowledge about the mechanism of ajoene action, a study on ajoene antibacterial effect in $M$. tuberculosis suggested ajoene-induced stress response in the endoplasmic reticulum (ER), 
which represents part of the defense mechanism of the host against Mycobacterial infection [189].

Ajoene promotes reactive oxygen species (ROS) production through activation of c-Jun $\mathrm{N}$-terminal kinase (JNK). Upon JNK activation, ROS production increased, resulting in the activation of ER stress and autophagy [189]. In another study, ajoene exhibited quorum sensor (QS) inhibiting activity, a system used by $P$. aeruginosa to synchronize for specific genes included in its pathogenicity [191]. A synthetic ajoene was used in both in vitro and in vivo models by the previous study. DNA microarray assay indicated that synthetic ajoene inhibited QS in a dose-dependent manner. Furthermore, it has biofilm killing property, which accounts for ajoene synergy with antibiotics such as tobramycin [191].

Antimicrobial peptides (AMPs) are a group of peptides that play a crucial role in the host defense mechanism [192]. AMPs occur in a variety of microorganisms, animals, and plant kingdoms [192]. With the emergence of antibiotic resistance, several strategies are proposed to tackle this problem, like using plant antimicrobial peptides from plants (PAMPs) as an alternative to antibiotics [193]. PAMPs are cysteine-rich cationic compounds (31) classified into six families according to cysteine number, amino acid chain, and disulfide bond contents: thionins, defensins, haveins, lipid transfer proteins, cyclotides, and snakins [194]. These PAMPs represent plant defense barriers against pathogens isolated from roots, flowers, seeds, stems, and leaves of several species [194]. In a recent study, PAMP was isolated from leaves of Trianthema portulacastrum L. and tested for antimicrobial activity toward MDR bacterial strains [193]. PAMP significantly inhibits the growth of $S$. aureus and B. subtilis, but it showed no activity toward E. coli. Further assessment is needed to identify the mechanism behind this action [193].

3.4. Terpenes. Terpenes are a large group of diverse organic molecules produced by various plants and animals, which are essential for their survival [195, 196]. Also, terpenes possess pharmacological and biological properties beneficial to humans. Chemically, terpenes are hydrocarbons with a 5carbon isoprene unit as the main building block for their biosynthesis [197]. According to the number of isoprenes, terpenes are classified into monoterpenes (e.g., carvone, geraniol, and D-limonene), which account for $67 \%$ of biologically active terpenes [198], diterpenes (e.g., retinol and retinoic acid), triterpenes (e.g., betulinic acid and oleanolic acid), and tetraterpenes (e.g., $\alpha$-carotene, $\beta$-carotene, and lutein) [199] (Figure 7 illustrates the chemical structures of selected terpenes with antimicrobial activity toward MDR bacteria).

Monoterpenes are volatile compounds representing the main constituents of plant's essential oil and are frequently used in fragrance and aromatherapy [199]. Several studies claimed the broad antimicrobial activity of carvacrol (32), thymol (33), eugenol (34), and menthol (35) through efflux pump inhibition and inhibition of bacterial growth and membrane properties as well, with thymol being the most active toward S. aureus and E. coli [197, 200, 201]. Geraniol (36) exhibits significant activity against resistant Gramnegative pathogens such as Enterobacter species through efflux pump inhibition [202].

In another study, the effect of four monoterpenes on the biomembrane of $S$. aureus and E. coli were assessed through differential scanning calorimetry (DSC) technique [203]. This study tested the interaction between thymol, carvacrol, p-cymene, and gamma terpenes with dimyristoylphosphatidylcholine vehicle. The result indicated that thymol has a bactericidal effect on $S$. aureus. In contrast, carvacrol and p-cymene have an inhibitory action on E. coli. Both produce their effects in a concentration-dependent manner. Furthermore, the study suggested that disturbance of membrane lipid is the possible mechanism of action for these monoterpenes toward MDR pathogens, and the effect is highly dependent on lipid contents of the bacterial membrane [203].

Similarly, Garcia-salinas et al. conducted a study to demonstrate the effect of essential oils (EOs) from plants on the membrane properties of $S$. aureus and E. coli [204]. Carvacrol, cinnamaldehyde, and thymol were used in this study with chlorhexidine $(0.004 \mathrm{mg} / \mathrm{mL})$ as reference. SEM, flow cytometry, and confocal microscopy results indicated that these compounds effectively inhibit bacterial growth in vitro through disruption of membrane integrity. In addition, carvacrol, thymol, and cinnamaldehyde may affect bacterial biofilm formation in concentration equal or more than $0.5 \mathrm{mg} / \mathrm{mL}$. It was suggested that the hydroxyl group in carvacrol and thymol has a role in this mechanism by inactivating microbial enzymes and interacting with the cell membrane. As a consequence, the leakage of intracellular components would be increased [204].

Retinol (vitamin A) (37) and its active form retinoic acid (38) are another class of terpenes present in carrots, sweet potatoes, pumpkin, squash, and cantaloupe [205]. Skin is the main target of these compounds, and several studies confirmed their activity in treating skin-related infectious diseases [206, 207]. As a mechanism, vitamin A contributes to gene expression through binding into two types of receptors-retinoic acid receptors (RAR) and retinoid $X$ receptors ( $R X R$ ) - which are abundant in the skin [208]. A new synthetic class of retinoid CD437 (39) was evaluated for potential bactericidal effect toward E. faecalis [209]. CD437 reported anti-biofilm activity toward this pathogen and profound synergy when combined with gentamycin [209]. In contrast, another study suggested that CD437 bacterial-killing property was linked to disruption of the lipid membrane [210]. In this study, two synthetic retinoids CD437 and CD1530 were tested for bactericidal action in MRSA and S. aureus infections.

Molecular dynamics simulations indicated that these compounds successfully penetrate and establish bacterial lipid bilayers. Moreover, these compounds reveal a significant synergistic effect with gentamycin against MRSA in mouse-model [210]. Triterpenes are a large, diverse group of phytochemicals with more than 20,000 compounds identified and recognized for broad pharmacological properties 
<smiles>Cc1ccc(C(C)C)cc1O</smiles>

[32]<smiles>Cc1ccc(C(C)C)c(O)c1</smiles>

[33]<smiles>C=CCc1ccc(O)c(OC)c1</smiles>

[34]<smiles>CC1CCC(C(C)C)C(O)C1</smiles>

[35]<smiles>CC(C)=CCCC(C)=CCO</smiles>

[36]<smiles>CC1=C(/C=C/C(C)=C/C=C/C(C)=C/CO)C(C)(C)CCC1</smiles>

[37]<smiles>O=C(O)c1ccc2cc(-c3ccc(O)c(C45CC6CC(CC(C6)C4)C5)c3)ccc2c1</smiles>

[39]<smiles>CC1=C(/C=C/C(C)=C/C=C/C(C)=C/C(=O)O)C(C)(C)CCC1</smiles>

[38]

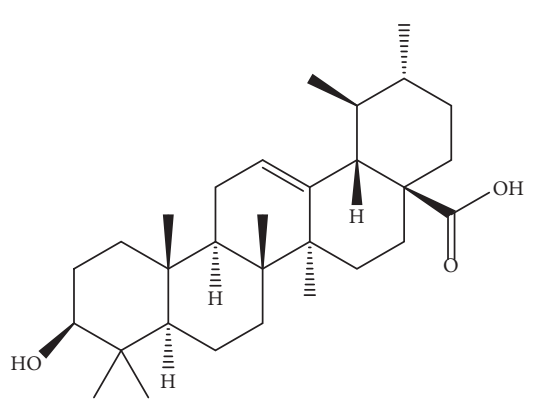

[41]

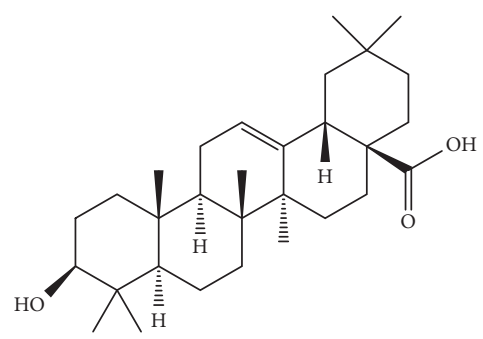

[40]

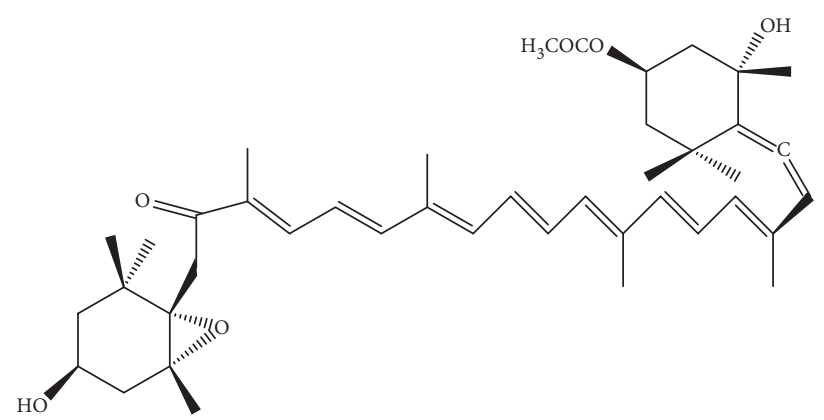

[43]

FIgURE 7: The chemical structures of selected terpenes reported with antimicrobial activity against MDR bacteria. 
like anti-inflammatory, analgesic, cardiotonic, sedative, antimicrobial, and anticancer [211, 212].

Oleanolic acid (OA) (40) is triterpene isolated from 2000 plant species, mainly plants belonging to Oleaceae family such as the Olea europaea (olive), and functions as a barrier to protect the plant from water loss and pathogens [213]. Several studies have proven OA's efficacy and its derivatives as antimicrobial agents (MIC $\leq 100 \mu \mathrm{g} / \mathrm{mL})$ against S. pneumoniae, E. faecalis, $P$. aeruginosa, M. tuberculosis, Streptococci, and E. coli [211, 214-216]. Although there is little knowledge about OA action's mechanism, some studies proposed induction of stress response and efflux pump inhibition as primary targets for this class [211].

Ursolic acid (UA) (41), which is another triterpenoid acid, shares most features in common with oleanolic acid (OA) [211]. It is abundant in the peel of berries, especially cranberries. Kurek et al. studied the mechanism of OA and UA action toward Gram-positive Listeria monocytogenes [217]. It was shown that both terpenoid acids reduce listeriolysin $\mathrm{O}$ hemolytic activity without influencing its synthesis or function. Besides, these compounds affect the biofilm formation of Listeria monocytogenes; however, the reason for such activity is still unclear and demands further clarification [217]. In another study, 3-O- $\alpha$-L-arbinopyranoside (URS) (42), an ursolic acid phenotype isolated from leaves of Acanthopanax henryi (Oliv.) Harms., was evaluated for its antimicrobial potential against MRSA alone and in combination with oxacillin [218]. MIC $(6.25 \mu \mathrm{g} / \mathrm{mL})$, timekill assay, white checkboard dilution test, and transmission electron microscopy (TEM) results indicated that URS has significant antimicrobial activity toward MRSA through inhibition of bacterial cell wall and induction of cell lysis mechanism. URS showed partial synergy with oxacillin, resulting in the suppression of the time-kill growth curve below the inhibitory level [218].

Carotenoids are natural pigments produced by several organisms such as bacteria, yeast, algae, molds, and plants with valuable physiological and pharmacological properties [219]. They acquired importance as a precursor for vitamin $\mathrm{A}$ as well as their potential as antioxidant and anticancer agents [220]. Carotenoids are classified into two groups: hydrocarbon carotenes (e.g., $\beta$-carotene, torulene) and oxygenated xanthophylls (e.g., astaxanthin, torularhodin) [220]. Few studies were performed to confirm the antimicrobial activity of carotenoids; among them is a study on fucoxanthin (43) carotenoids produced by algae [221]. According to the study, fucoxanthin showed promising antimicrobial effects toward Gram-positive and Gramnegative bacteria, including $S$. aureus (MIC $62.5 \mu \mathrm{g} / \mathrm{mL}$ ) [221]. However, further studies to demonstrate the possible mode of action is required.

Spathulenol, 1,8-cineole, trans-caryophyllene, $\beta$-pinene, $\beta$-eudesmol, camphor, $\alpha$-pinene, and caryophyllene oxide are essential oils isolated from the leaves and flowers of Salvia hydrangea DC. ex Benth., an Iranian shrub that belongs to the Lamiaceae family. This plant has been used in traditional medicine to treat various bacterial and fungal infections. In the study, the isolated oils exhibited a significant inhibitory and lethal effect toward Gram-negative bacteria $P$. aeruginosa (MIC $\sim 16 \mu \mathrm{g} / \mathrm{mL}$ ) and $K$. pneumoniae (MIC $\sim 62 \mu \mathrm{g} / \mathrm{mL})$ [222]. These results highlighted the potential application of essential oils to develop new therapeutic agents in the future.

3.5. Coumarins. Coumarins are a group of natural products identified as secondary metabolites in plants, fungi, and bacteria with vast biological properties such as anti-inflammatory, anti-coagulant, antiviral, antibacterial, antifungal, vasodilation, anticancer, antihypertensive, antioxidant, and neuroprotective actions [223]. Coumarin is distributed to all parts of the plant, particularly in fruit (e.g., Aegle marmelos (L.) Corrêa., Tetrapleura tetraptera (Schum. \& Thonn.) Taub.) [224], seeds (e.g., Calophyllum cerasiferum Vesque, Calophyllum inophyllum L.) [225], root (e.g., Ferulago campestris (Besser) Grecescu.) [226], and leaves (e.g., Murraya paniculata (L.) Jack.) [227].

Based on chemical structure, coumarins split into six subgroups: simple coumarins, furanocoumarins, dihydrofurano coumarins, pyrano coumarins (linear and angular types), phenyl coumarins, and bicoumarins [223]. Many studies manifested the antimicrobial activity of both natural and synthetic coumarins toward MDR bacteria. For instance, six coumarins isolated from the aerial part of Rhododendron lepidotum Wall. ex G. Don. exhibited considerable antimicrobial activity toward MRSA ATCC15187, E. coli ATCC-8739, P. aeruginosa ATCC-9027, with daphnetin (44) compound being the most active coumarin (MIC $125 \mu \mathrm{g} / \mathrm{mL}$ ) compared to ciprofloxacin reference drug [228] (Figure 8 illustrates selected coumarins with antimicrobial activity toward MDR bacteria).

In another study, disesquiterpene (45) coumarin obtained from the root of Ferula pseudalliacea Rech.f. showed higher antibacterial activity toward Gram-positive pathogens [229]. In this study, disesquiterpene activity was tested against $S$. aureus ATCC 25,922, VRE, E. coli ATCC25,922, $P$. aeruginosa PTCC1430, and $K$. pneumoniae in a concentration equal to $64 \mu \mathrm{g} / \mathrm{mL}$ [229]. Moreover, asphodelin A (46), which is an aryl coumarin from Asphodelus microcarpus, exhibited potent antimicrobial activity (MIC $4-128 \mu \mathrm{g} / \mathrm{mL}$ ) toward S. aureus, E. coli, and P. aeruginosa, whereas the glycoside form asphodelin A $4^{\prime}-\mathrm{O}-\beta$-D-glycoside showed moderate activity [230]. Reports in the structural activity relationship (SAR) of coumarins suggested that lipophilic properties and planar structure are important for antibacterial effect as the mechanism of coumarin action involves cellular penetration through passive diffusion [231]. In addition, the SAR of three coumarins derived from Streptomyces species indicated that the sugar moiety of coumarin is essential for biological activity [232]. It is also suggested that inhibition of DNA gyrase enzyme is the mode of action of these coumarins [232].

Another study in the coumarin mechanism of action proposed that coumarin suppresses quorum-sensing activity, decreasing bacterial virulence and biofilm formation [233]. Previous reports indicated that oxidative stress plays a crucial role in antibiotic resistance [234, 235]. Thus, it is better to look for alternative medication with dual 


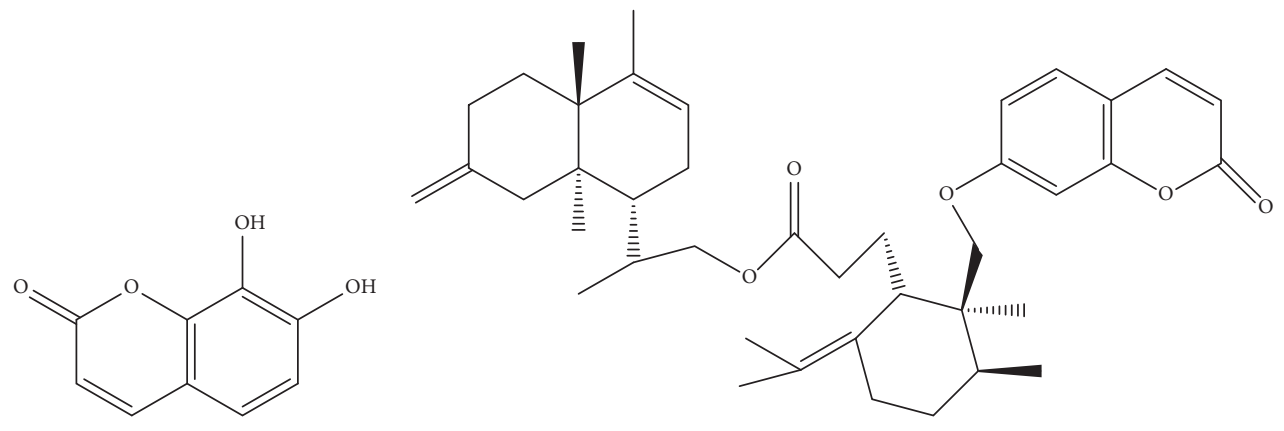

[44]

[45]<smiles>O=c1oc2cc(O)ccc2c(O)c1-c1ccc(O)cc1O</smiles>

[46]<smiles>CC(=O)c1ccc(-n2cc(-c3cc4oc(=O)ccc4cc3C(C)=O)nn2)cc1</smiles>

[48]<smiles>O=c1oc2ccccc2c(O)c1/N=N/c1ccc(Cl)cc1</smiles>

[50]<smiles>O=c1oc2cc(O)c(O)cc2cc1-c1ccccc1</smiles>

[47]<smiles>O=C1C(=O)N(CCn2cc(COc3cc(=O)oc4ccccc34)nn2)c2ccccc21</smiles>

[49]

FIGURE 8: Selected coumarins with antimicrobial activity toward MDR bacteria.

antibacterial and antioxidant activities. In accordance, a series of hydroxy-3-aryl coumarins were synthesized and screened for antibacterial and antioxidant activity toward MDR bacteria [236]. Docking studies were performed as well to determine the most active compound in this series. 3phenyl coumarin with hydroxyl substitution at positions 5 and 7 (47) exhibited higher activity against $S$. aureus (MIC $11 \mu \mathrm{g} / \mathrm{mL}$ ), followed by MRSA (MIC $22 \mu \mathrm{g} / \mathrm{mL}$ ). Furthermore, a docking study suggested that tyrosyl-tRNA synthase, topoisomerase II, and DNA gyrase are the most appropriate binding sites in the bacterial target [236].
New series of coumarin triazole derivatives were synthesized and studied for in vitro antibacterial activity as well as binding properties to MurB protein [237]. It was noticed that substitution in position 6 or 7 of coumarin ring with 4carboxyphenyltriazolyl (48) markedly enhance the antimicrobial activity toward $S$. aureus (MIC $0.16-6.28 \mu \mathrm{g} / \mathrm{mL}$ ) while bromine or aryl substitution in position 3 account for higher selectivity toward E. coli (MIC $0.02-0.15 \mu \mathrm{g} / \mathrm{mL}$ ) [237]. Similarly, other coumarin derivatives with indolinedione (49) were synthesized and studied for structural relationship on the antimicrobial activity against various 
Gram-positive and Gram-negative strains, including E. coli and $S$. aureus [238].

The SAR study established that the electronic environment of indolinedione positively affected the antimicrobial activity of these compounds, and the activity considerably reduced when the chain length between triazole and indolinedione portion increased. As a mechanism, docking studies suggested that binding to $S$. aureus dihydrofolate reductase is the mode of action of these hybrid molecules [238]. Some literature indicated that the metal complex formation between transition metals like cobalt, nickel, zinc, copper, and drug molecule significantly improves the drug's biological function $[239,240]$. As an explanation, the metal complex serves as a vehicle for drug ligand, and the bond between the metal and ligand cleaves easily inside the body leading to accelerated drug action and increased efficacy [241, 242].

This approach is widely investigated to enhance antimicrobial, antifungal, and anticancer activities [243]. For instance, chromen-2-one complex of 3-aryl-azo-4-hydroxy coumarin (50) exhibited excellent antimicrobial activity toward E. coli (MTCC 614), K. pneumoniae (MTCC 109), and $S$. aureus with MIC equal to $31.25 \mu \mathrm{g} / \mathrm{mL}$ [240]. In a recent study, a new coumarin compound 3,3'-(3,4dichlorobenzylidene)-bis-(4-hydroxycoumarin) was detected as an inhibitor to MRSA biofilm formation. Molecular docking study suggested that the anti-MRSA effect is due to targeting bacterial arginine repressor (ArgR), a property that makes this compound a promising source for the development of new anti-MRSA agents [244, 245].

\section{Conclusions and Future Perspectives}

Nature represents an abundant source for bioactive compounds that are available, affordable, and easy to isolate with almost no harm to humans. Plant-derived compounds have been used since ancient times to treat various infectious diseases. For instance, honey is used as an ointment since the medieval era to treat wounds and prevent secondary infections. Also, Echinacea extract was used by native Americans for the same purpose. With the rapid progression of bacterial resistance to conventional antibiotics, scientists returned to nature, searching for alternatives. Many studies had been done, and the therapeutic potential of plant-derived compounds has been documented. Some of these compounds are active as antibiotics when they are used alone while others may enhance the antibacterial action when coadministered with existing antibiotics. Thus, combination therapy of classical antibiotics with phytochemicals could be a promising solution to overcome resistance. However, designing new medication from the plant is still challenging.

Various mechanisms have been proposed to explain plant-derived compounds' action, including efflux pump inhibition, destruction of the bacterial membrane, inhibition of biofilm formation, DNA, and protein synthesis. Understanding the specific mechanism behind phytochemical action is vital to develop a new antimicrobial agent. Nevertheless, shifting from in vitro study to in vivo study and clinical trial remains a big confrontation since several factors may affect the efficacy of phytochemicals like tissue penetration and bioavailability. As such, a better understanding of pharmacokinetic and pharmacodynamic of the natural molecule is necessary.

$\begin{array}{ll}\text { Abbreviations } \\ \text { MDR: } & \text { Multidrug resistance } \\ \text { EP: } & \text { Efflux pump } \\ \text { E. coli: } & \text { Escherichia coli } \\ \text { MRSA: } & \text { Methicillin-resistant Staphylococcus aureus } \\ \text { K. pneumoniae: } & \text { Klebsiella pneumoniae } \\ \text { P. aeruginosa: } & \text { Pseudomonas aeruginosa } \\ \text { VRE: } & \text { Vancomycin-resistant Enterococci } \\ \text { VRSA: } & \text { Vancomycin-resistant Staphylococcus } \\ & \text { aureus } \\ \text { VISA: } & \text { Vancomycin-intermediate Staphylococcus } \\ & \text { aureus } \\ \text { CRE: } & \text { Carbapenem-resistant Enterobacteriaceae } \\ \text { MIC: } & \text { Minimum inhibitory concentration } \\ \text { RND: } & \text { Resistance-nodulation family } \\ \text { SMR: } & \text { Small multidrug resistance } \\ \text { MFS: } & \text { Major facilitator superfamily } \\ \text { MATE: } & \text { Multidrug and toxin extrusion } \\ \text { ABC: } & \text { Adenosine triphosphate binding cassette. }\end{array}$

\section{Data Availability}

No data were used to support this study.

\section{Conflicts of Interest}

The authors declare no conflicts of interest.

\section{References}

[1] A. Chokshi, Z. Sifri, D. Cennimo, and H. Horng, "Global contributors to antibiotic resistance," Journal of Global Infectious Diseases, vol. 11, no. 1, pp. 36-42, 2019.

[2] W. Li, H. Zhang, Y. G. Assaraf et al., "Overcoming ABC transporter-mediated multidrug resistance: molecular mechanisms and novel therapeutic drug strategies," Drug Resistance Updates, vol. 27, 2016.

[3] R. Adrizain, F. Suryaningrat, A. Alam, and D. Setiabudi, "Incidence of multidrug-resistant, extensively drug-resistant and pan-drug-resistant bacteria in children hospitalized at Dr. Hasan Sadikin general hospital Bandung Indonesia," IOP Conference Series: Earth and Environmental Science, vol. 125, no. 1, pp. 6-10, 2018.

[4] World Health Organization, Global Tuberculosis Report 2014, World Health Organization, Geneva, Switzerland, 2014.

[5] CDC, Antibiotic Resistance Threats in the United States, U.S. Department of Health and Human Services, CDC, Atlanta, GA, USA, 2019.

[6] J. O. Neill, Antimicrobial Resistance: Tackling a Crisis for the Health and Wealth of Nations the Review on Antimicrobial Resistance Chaired, World Health Organization, London, UK, 2014.

[7] U. Okwu, M. Olley, O. Akpoka, and E. Izevbuwa, "Methicillin-resistant Staphylococcus aureus (MRSA) and anti- 
MRSA activities of extracts of some medicinal plants: a brief review," AIMS Microbiology, vol. 5, no. 2, pp. 117-137, 2019.

[8] A.-P. Magiorakos, A. Srinivasan, R. B. Carey et al., "Multidrug-resistant, extensively drug-resistant and pandrugresistant bacteria: an international expert proposal for interim standard definitions for acquired resistance," Clinical Microbiology and Infection, vol. 18, no. 3, pp. 268-281, 2012.

[9] S. Basak, P. Singh, and M. Rajurkar, "Multidrug resistant and extensively drug resistant bacteria: a study," Journal of Pathogens, vol. 2016, Article ID 4065603, 5 pages, 2016.

[10] T. H. Holtz, "XDR-TB in South Africa: revised definition," PLoS Medicine, vol. 4, no. 4, Article ID e161, 2007.

[11] D. van Duin and D. L. Paterson, "Multidrug-resistant bacteria in the community: trends and lessons learned," Infectious disease clinics of North America, vol. 30, no. 2, pp. 377-390, 2016.

[12] Y. Haibo, "Naxi students' national identity construction and schooling: a case study of Lijiang no.1 senior secondary school," China: An International Journal, vol. 7, no. 1, pp. 161-175, 2009.

[13] G. F. Ferrazzano, T. Cantile, L. Roberto et al., "Determination of the in vitro and in vivo antimicrobial activity on salivary Streptococci and Lactobacilli and chemical characterisation of the phenolic content of a Plantago lanceolata infusion," BioMed Research International, vol. 2015, Article ID 286817, 8 pages, 2015.

[14] H. O. Lawal, S. O. Etatuvie, and A. B. Fawehinmi, "Ethnomedicinal and pharmacological properties of Morinda lucida," Journal of Natural Products, vol. 5, pp. 93-98, 2012.

[15] C. O. Nwonuma, T. A. Adelani-Akande, O. O. Osemwegie, A. F. Olaniran, and T. A. Adeyemo, "Comparative study of in vitro antimicrobial potential and phytochemicals of some medical plants," F1000Research, vol. 8, p. 81, 2019.

[16] Z. Shewamene, T. Dune, and C. A. Smith, "Use of traditional and complementary medicine for maternal health and wellbeing by African migrant women in Australia: a mixed method study," BMC Complementary Medicine and Therapies, vol. 20, no. 1, p. 60, 2020.

[17] M. J. Cheesman, A. Ilanko, B. Blonk, and I. E. Cock, "Developing new antimicrobial therapies: are synergistic combinations of plant extracts/compounds with conventional antibiotics the solution?" Pharmacognosy Reviews, vol. 11, no. 22, pp. 57-72, 2017.

[18] S. Manandhar, S. Luitel, and R. K. Dahal, "In vitro antimicrobial activity of some medicinal plants against human pathogenic bacteria," Journal of Tropical Medicine, vol. 2019, Article ID 1895340, 5 pages, 2019.

[19] A. T. Al-Sa'ady, "Antibacterial screening for five local medicinal plants against nosocomial pathogens: Klebsiella pneumoniae and Staphylococcus epidermidis," EurAsian Journal of BioSciences, vol. 14, no. 1, pp. 553-555, 2020.

[20] R. Gobalakrishnan, R. Bhuvaneswari, and M. Rajkumar, "Natural antimicrobial and bioactive compounds from Ludwigia parviflora Roxb." Journal of Analytical \& Pharmaceutical Research, vol. 9, no. 1, pp. 37-42, 2020.

[21] B. Aslam, W. Wang, M. I. Arshad et al., "Antibiotic resistance: a rundown of a global crisis," Infection and Drug Resistance, vol. 11, pp. 1645-1658, 2018.

[22] G. M. Rossolini, F. Arena, P. Pecile, and S. Pollini, "Update on the antibiotic resistance crisis," Current Opinion in Pharmacology, vol. 18, pp. 56-60, 2014.

[23] P. D. Gupta and T. J. Birdi, "Development of botanicals to combat antibiotic resistance," Journal of Ayurveda and Integrative Medicine, vol. 8, no. 4, pp. 266-275, 2017.
[24] A. V. Vargiu, K. M. Pos, K. Poole, and H. Nikaido, "Editorial: bad bugs in the XXIst century: resistance mediated by multidrug efflux pumps in gram-negative bacteria," Frontiers in Microbiology, vol. 7, 2016.

[25] B. Khameneh, M. Iranshahy, V. Soheili, and B. S. Fazly Bazzaz, "Review on plant antimicrobials: a mechanistic viewpoint," Antimicrobial Resistance and Infection Control, vol. 8, no. 1, p. 118, 2019.

[26] I. Ghai and S. Ghai, "Understanding antibiotic resistance via outer membrane permeability," Infection and Drug Resistance, vol. 11, pp. 523-530, 2018.

[27] A. Witzky, R. Tollerson, and M. Ibba, "Translational control of antibiotic resistance,” Open Biology, vol. 9, no. 7, Article ID 190051, 2019.

[28] P. Blanco, S. Hernando-Amado, J. Reales-Calderon et al., "Bacterial multidrug efflux pumps: much more than antibiotic resistance determinants," Microorganisms, vol. 4, no. 1, p. 14, 2016.

[29] L. McMurry, R. E. Petrucci, and S. B. Levy, "Active efflux of tetracycline encoded by four genetically different tetracyline resistance determinants in Escherichia coli," Proceedings of the National Academy of Sciences of the United States of America, vol. 77, no. 7 II, pp. 3974-3977, 1980.

[30] H. Nikaido, "Structure and mechanism of RND-type multidrug efflux pumps," Advances in Enzymology and Related Areas of Molecular Biology, vol. 77, no. 11, pp. 1-60, 2010.

[31] Y. Liu, Y. Liu, L. He, Y. Zhao, and X. C. Zhang, "Singlemolecule fluorescence studies on the conformational change of the ABC transporter MsbA," Biophysics Reports, vol. 4, no. 3, pp. 153-165, 2018.

[32] T. Kusakizako, H. Miyauchi, R. Ishitani, and O. Nureki, "Structural biology of the multidrug and toxic compound extrusion superfamily transporters," Biochimica et Biophysica Acta (BBA)-Biomembranes, vol. 1862, no. 12, Article ID 183154, 2020.

[33] B. J. Willson, L. Dalzell, L. N. M. Chapman, and G. H. Thomas, "Enhanced functionalisation of major facilitator superfamily transporters via fusion of C-terminal protein domains is both extensive and varied in bacteria," Microbiology, vol. 165, no. 4, pp. 419-424, 2019.

[34] V. Ovchinnikov, T. A. Stone, C. M. Deber, and M. Karplus, "Structure of theEmrEmultidrug transporter and its use for inhibitor peptide design," Proceedings of the National Academy of Sciences, vol. 115, no. 34, pp. E7932-E7941, 2018.

[35] I. Sultan, S. Rahman, A. T. Jan, M. T. Siddiqui, A. H. Mondal, and Q. M. R. Haq, "Antibiotics, resistome and resistance mechanisms: a bacterial perspective," Frontiers in Microbiology, vol. 9, 2018.

[36] J. Dreier and P. Ruggerone, "Interaction of antibacterial compounds with RND efflux pumps in Pseudomonas aeruginosa," Frontiers in Microbiology, vol. 6, pp. 1-21, 2015.

[37] M. R. Schroeder and D. S. Stephens, "Macrolide resistance in Streptococcus pneumoniae," Frontiers in Cellular and Infection Microbiology, vol. 1-9, 2016.

[38] S. N. Abdi, R. Ghotaslou, K. Ganbarov et al., "Acinetobacter baumannii efflux pumps and antibiotic resistance," Infection and Drug Resistance, vol. 13, pp. 423-434, 2020.

[39] U. Choi and C. R. Lee, "Distinct roles of outer membrane porins in antibiotic resistance and membrane integrity in Escherichia coli," Frontiers in Microbiology, vol. 10, 2019.

[40] K. Phan and T. Ferenci, "A design-constraint trade-off underpins the diversity in ecologically important traits in species Escherichia coli," The ISME Journal, vol. 7, no. 10, pp. 2034-2043, 2013. 
[41] M. Sato, K. Machida, E. Arikado, H. Saito, T. Kakegawa, and H. Kobayashi, "Expression of outer membrane proteins in Escherichia coli growing at acid $\mathrm{pH}$," Applied and Environmental Microbiology, vol. 66, no. 3, pp. 943-947, 2000.

[42] L. Fernández and R. E. W. Hancock, "Adaptive and mutational resistance: role of porins and efflux pumps in drug resistance," Clinical Microbiology Reviews, vol. 25, no. 4, pp. 661-681, 2012.

[43] B. K. Ziervogel and B. Roux, "The binding of antibiotics in OmpF porin," Structure, vol. 21, no. 1, pp. 76-87, 2013.

[44] E. Sugawara, S. Kojima, and H. Nikaido, "Klebsiella pneumoniae major porins OmpK35 and OmpK36 allow more efficient diffusion of $\beta$-lactams than their Escherichia coli homologs OmpF and OmpC," Journal of Bacteriology, vol. 198 , no. 23, pp. 3200-3208, 2016.

[45] S. Chevalier, E. Bouffartigues, J. Bodilis et al., "Structure, function and regulation of Pseudomonas aeruginosa porins," FEMS Microbiology Reviews, vol. 41, no. 5, pp. 698-722, 2017.

[46] B. Jubeh, Z. Breijyeh, and R. Karaman, "Resistance of grampositive bacteria to current antibacterial agents and overcoming approaches," Molecules, vol. 25, no. 12, 2020.

[47] Z. Pang, R. Raudonis, B. R. Glick, T.-J. Lin, and Z. Cheng, "Antibiotic resistance in Pseudomonas aeruginosa: mechanisms and alternative therapeutic strategies," Biotechnology Advances, vol. 37, no. 1, pp. 177-192, 2019.

[48] A. M. Egorov, M. M. Ulyashova, and M. Y. Rubtsova, "Bacterial enzymes and antibiotic resistance," Acta Naturae, vol. 10, no. 4, pp. 33-48, 2018.

[49] G. Wright, "Bacterial resistance to antibiotics: enzymatic degradation and modification," Advanced Drug Delivery Reviews, vol. 57, no. 10, pp. 1451-1470, 2005.

[50] J. Lin, D. Zhou, T. A. Steitz, Y. S. Polikanov, and M. G. Gagnon, "Ribosome-targeting antibiotics: modes of action, mechanisms of resistance, and implications for drug design," Annual Review of Biochemistry, vol. 87, no. 1, pp. 451-478, 2018.

[51] R. K. Upadhya, L. Shenoy, and R. Venkateswaran, "Effect of intravenous dexmedetomidine administered as bolus or as bolus-plus-infusion on subarachnoid anesthesia with hyperbaric bupivacaine," Journal of Anaesthesiology, Clinical Pharmacology, vol. 34, no. 3, pp. 46-50, 2018.

[52] K. Bush, "Past and present perspectives on $\beta$-lactamases," Antimicrobial Agents and Chemotherapy, vol. 62, no. 10, 2018.

[53] P. Bhattacharya, M. Singha, K. Senapati et al., "Chloramphenicol-borate/boronate complex for controlling infections by chloramphenicol-resistant bacteria," RSC Advances, vol. 8, no. 32, pp. 18016-18022, 2018.

[54] K. M. Belaynehe, S. W. Shin, P. Hong-Tae, and H. S. Yoo, "Occurrence of aminoglycoside-modifying enzymes among isolates of Escherichia coli exhibiting high levels of aminoglycoside resistance isolated from Korean cattle farms," FEMS Microbiology Letters, vol. 364, no. 14, pp. 1-9, 2017.

[55] D. Jelić and R. Antolović, "From erythromycin to azithromycin and new potential ribosome-binding antimicrobials," Antibiotics (Basel, Switzerland), vol. 5, no. 3, 2016.

[56] M. Goudarzi and M. Navidinia, "Overview perspective of bacterial strategies of resistance to biocides and antibiotics," Archives of Clinical Infectious Diseases, vol. 14, no. 2, 2019.

[57] P. E. Akpaka, S. Kissoon, C. Wilson, P. Jayaratne, A. Smith, and G. R. Golding, "Molecular characterization of vancomycin-resistant Enterococcus faecium isolates from
Bermuda," PLoS One, vol. 12, no. 3, pp. 1-9, Article ID e0171317, 2017.

[58] A. Savoldi, E. Carrara, D. Y. Graham, M. Conti, and E. Tacconelli, "Prevalence of antibiotic resistance in Helicobacter pylori: a systematic review and meta-analysis in world health organization regions," Gastroenterology, vol. 155, no. 5, pp. 1372-1382, 2018.

[59] C. M. Oliphant and K. Eroschenko, "Antibiotic resistance, part 1: gram-positive pathogens," The Journal for Nurse Practitioners, vol. 11, no. 1, pp. 70-78, 2015.

[60] P. Pachori, R. Gothalwal, and P. Gandhi, "Emergence of antibiotic resistance Pseudomonas aeruginosa in intensive care unit; a critical review," Genes \& Diseases, vol. 6, no. 2, pp. 109-119, 2019.

[61] C. Cillóniz, C. Dominedò, and A. Torres, "Multidrug resistant gram-negative bacteria in community-acquired pneumonia," Critical Care (London, England), vol. 23, no. 1, p. 79, 2019.

[62] J. V. Ashurst and A. Dawson, Klebsiella Pneumonia Treatment/Management, Vol. 6-9, Medscape, New York, NY, USA, 2019.

[63] A. Melese, C. Genet, and T. Andualem, "Prevalence of vancomycin resistant Enterococci (VRE) in Ethiopia: a systematic review and meta-analysis," BMC Infectious Diseases, vol. 20, no. 1, p. 124, 2020.

[64] F. Del Bianco, M. Morotti, S. Zannoli et al., "Comparison of four commercial screening assays for the detection of blakpc, blandm, blaimp, blavim, and blaoxa48 in rectal secretion collected by swabs," Microorganisms, vol. 7, no. 12, 2019.

[65] A. Lamut, L. Peterlin Mašič, D. Kikelj, and T. Tomašič, "Efflux pump inhibitors of clinically relevant multidrug resistant bacteria," Medicinal Research Reviews, vol. 39, no. 6, pp. 2460-2504, 2019.

[66] C. Jain, S. Khatana, and R. Vijayvergia, "Bioactivity of secondary metabolites of various plants: a review," International Journal of Pharmaceutical Sciences and Research, vol. 10, no. 2, pp. 494-504, 2019.

[67] L. Maman Manzo, I. Moussa, K. Ikhiri, and L. Yu, “Toxicity studies of Acacia nilotica (L.): a review of the published scientific literature Implication for health policy/practice/ research/medical education," Journal of HerbMed Pharmacology, vol. 8, no. 3, 2019.

[68] R. P. Samy, P. N. Pushparaj, and P. Gopalakrishnakone, "A compilation of bioactive compounds from ayurveda," Bioinformation, vol. 3, no. 3, pp. 100-110, 2008.

[69] A. Hemavathy, P. Shanthi, C. Sowndharya, S. Thiripura Sundari, and K. Priyadharshni, "Extraction and isolation of bioactive compounds from a therapeutic medicinal plant Wrightia tinctoria ( Roxb.) R. Br.” International Journal of Pharmacognosy and Phytochemical Research, vol. 11, no. 3, pp. 199-204, 2019.

[70] D. M. Pott, S. Osorio, and J. G. Vallarino, "From central to specialized metabolism: an overview of some secondary compounds derived from the primary metabolism for their role in conferring nutritional and organoleptic characteristics to fruit," Frontiers in Plant Science, vol. 10, p. 835, 2019.

[71] S. Pagare, M. Bhatia, N. Tripathi, S. Pagare, and Y. K. Bansal, "Secondary metabolites of plants and their role: overview," Current Trends in Biotechnology and Pharmacy, vol. 9, no. 3, pp. 293-304, 2015.

[72] J. C. Cardoso, M. E. B. d. Oliveira, and F. d. C. Cardoso, "Advances and challenges on the in vitro production of secondary metabolites from medicinal plants," Horticultura Brasileira, vol. 37, no. 2, pp. 124-132, 2019. 
[73] M. Dellagreca, A. Fiorentino, P. Monaco, L. Previtera, and A. Zarrelli, "A new dimeric 9,10-dihydrophenanthrenoid from the rhizome of Juncus acutus," Tetrahedron Letters, vol. 43, no. 14, pp. 2573-2575, 2002.

[74] M. DellaGreca, L. Previtera, R. Purcaro, and A. Zarrelli, "Cinnamic ester derivatives from oxalis pes-caprae (Bermuda buttercup)\#," Journal of Natural Products, vol. 70, no. 10, pp. 1664-1667, 2007.

[75] U. A. Khan, H. Rahman, M. Qasim et al., “Alkanna tinctoria leaves extracts: a prospective remedy against multidrug resistant human pathogenic bacteria," BMC Complementary and Alternative Medicine, vol. 15, no. 1, pp. 127-136, 2015.

[76] R. Khan, M. N. Baeshen, K. S. Saini, R. S. Bora, A. M. AlHejin, and N. A. Baeshen, "Antibacterial activities of Rhazya stricta leaf extracts against multidrug-resistant human pathogens," Biotechnology \& Biotechnological Equipment, vol. 30, no. 5, pp. 1016-1025, 2016.

[77] T. Siriyong, P. Srimanote, S. Chusri et al., "Conessine as a novel inhibitor of multidrug efflux pump systems in Pseudomonas aeruginosa," BMC Complementary and Alternative Medicine, vol. 17, no. 1, pp. 405-407, 2017.

[78] J. Reiter, N. Levina, M. Van Der Linden, M. Gruhlke, C. Martin, and A. J. Slusarenko, "Diallylthiosulfinate (allicin), a volatile antimicrobial from garlic (Allium sativum), kills human lung pathogenic bacteria, including MDR strains, as a vapor," Molecules (Basel, Switzerland), vol. 22, no. 10, pp. 1-14, 2017.

[79] J. K. Dzotam and V. Kuete, "Antibacterial and antibioticmodifying activity of methanol extracts from six Cameroonian food plants against multidrug-resistant enteric bacteria," BioMed Research International, vol. 2017, Article ID 1583510, 19 pages, 2017.

[80] N. M. Atef, S. M. Shanab, S. I. Negm, and Y. A. Abbas, "Evaluation of antimicrobial activity of some plant extracts against antibiotic susceptible and resistant bacterial strains causing wound infection," Bulletin of the National Research Centre, vol. 43, no. 1, p. 144, 2019.

[81] L. Padhi and S. K. Panda, "Antibacterial activity of Eleutherine bulbosa against multidrug-resistant bacteria," Journal of Acute Medicine, vol. 5, no. 3, pp. 53-61, 2015.

[82] B. Thapa, A. Singh, and R. Tuladhar, "In vitro antibacterial effect of medicinal plants against multidrug resistant gram negative bacteria," Tribhuvan University Journal of Microbiology, vol. 5, no. 1, pp. 25-31, 2018.

[83] M. F. Khan, H. Tang, J. T. Lyles, R. Pineau, Z. U. Mashwani, and C. L. Quave, "Antibacterial properties of medicinal plants from Pakistan against multidrug-resistant ESKAPE pathogens," Frontiers in Pharmacology, vol. 9, pp. 815-817, 2018.

[84] B. P. Marasini, P. Baral, P. Aryal et al., "Evaluation of antibacterial activity of some traditionally used medicinal plants against human pathogenic bacteria," BioMed Research International, vol. 2015, Article ID 265425, 6 pages, 2015.

[85] F. V. D. Assis, F. L. Siqueira, I. E. Gonçalves et al., "Antibacterial activity of lamiaceae plant extracts in clinical isolates of multidrug-resistant bacteria," Anais da Academia Brasileira de Ciências, vol. 90, no. 2, pp. 1665-1666, 2018.

[86] M. Masoumian and M. Zandi, "Antimicrobial activity of some medicinal plant extracts against multidrug resistant bacteria," Zahedan Journal of Research in Medical Sciences, vol. 19, no. 11, 2017.

[87] I. M. Famuyide, A. O. Aro, F. O. Fasina, J. N. Eloff, and L. J. McGaw, "Antibacterial activity and mode of action of acetone crude leaf extracts of under-investigated Syzygium and Eugenia (Myrtaceae) species on multidrug resistant porcine diarrhoeagenic Escherichia coli," BMC Veterinary Research, vol. 15, no. 1, p. 162, 2019.

[88] M. Goudarzi and H. Azimi, "Antimicrobial activity of Peganum harmala against methicillin-resistant Staphylococcus aureus strains and assessment of its cytotoxicity effect on HEK-293 cells," International Journal of Infection, vol. 4, no. 4, Article ID e15592, 2017.

[89] A. A. Bello, Z. Samman Tahan, A. Kitaz, and B. Tiba, "Phytochemical screening and anti-multidrug resistant Pseudomonas aeruginosa of some fabaceae medicinal plants growing in Aleppo-Syria," International Journal of Pharmacognosy and Phytochemical Research, vol. 11, no. 3, pp. 91-97, 2019.

[90] B. Saleh, R. Hammoud, and A. Al-Mariri, "Antimicrobial activity of Ficus sycomorus L. (Moraceae) leaf and stem-bark extracts against multidrug resistant human pathogens," Herba Polonica, vol. 61, no. 1, pp. 39-49, 2015.

[91] M. Imran, M. Imran, and S. Khan, "Antibacterial activity of Syzigium cumini leaf extracts against multidrug resistant pathogenic bacteria," Journal of Applied Pharmaceutical Science, vol. 7, no. 3, pp. 168-174, 2017.

[92] Y. H. Almawlah, H. Alaa, and S. O. Aljelawi, "Antibacterial activity of three plant extracts against multidrug resistance Pseudomonas aeruginosa," Asian Journal of Pharmaceutical and Clinical Research, vol. 10, no. 12, pp. 193-197, 2017.

[93] P. Agarwal, N. Agarwal, R. Gupta, M. Gupta, and B. Sharma, "Antibacterial activity of plants extracts against methicillinresistant Staphylococcus aureus and vancomycin-resistant Enterococcus faecalis," Journal of Microbial and Biochemical Technology, vol. 8, no. 5, pp. 404-407, 2016.

[94] I. K. Voukeng, V. P. Beng, and V. Kuete, "Antibacterial activity of six medicinal Cameroonian plants against grampositive and gram-negative multidrug resistant phenotypes," BMC Complementary and Alternative Medicine, vol. 16, no. 1 , p. $388,2016$.

[95] K. L. Shobha, A. S. Rao, K. S. R. Pai, and S. Bhat, "Antimicrobial activity of aqueous and ethanolic leaf extracts of Anacardium occidentale," Asian Journal of Pharmaceutical and Clinical Research, vol. 11, no. 12, pp. 474-476, 2018.

[96] L. Abdallah and G. Omar, "Antibacterial effect of some wild medicinal plants in Palestine against multidrug resistant Escherichia coli clinical isolate," Brazilian Journal of Biological Sciences, vol. 6, no. 12, pp. 103-113, 2019.

[97] D. Nigussie, G. Davey, B. A. Legesse, A. Fekadu, and E. Makonnen, "Antibacterial activity of methanol extracts of the leaves of three medicinal plants against selected bacteria isolated from wounds of lymphoedema patients," $B M C$ Complementary Medicine and Therapies, vol. 21, no. 1, p. 2, 2021.

[98] N. Srikacha and K. Ratananikom, "Antibacterial activity of plant extracts in different solvents against pathogenic bacteria: an in vitro experiment," Journal of Acute Disease, vol. 9, no. 5 , p. $223,2020$.

[99] F. J. Álvarez-Martínez, J. C. Rodríguez, F. Borrás-Rocher, E. Barrajón-Catalán, and V. Micol, "The antimicrobial capacity of Cistus salviifolius and Punica granatum plant extracts against clinical pathogens is related to their polyphenolic composition," Scientific Reports, vol. 11, no. 1, p. 588, 2021.

[100] J. Ribeiro, V. Silva, A. Aires, R. Carvalho, G. Igrejas, and P. Poeta, "Antimicrobial activity of phenolic compounds extracted from Platanus hybrida: exploring alternative 
therapies for a post-antibiotic era," Proceedings, vol. 66, no. 1, p. 18, 2021.

[101] E. H. Mohamed, Y. S. Alghamdi, S. Mostafa Abdel-Hafez et al., "Susceptibility assessment of multidrug resistant bacteria to natural products," Dose-response: A Publication of International Hormesis Society, vol. 18, no. 3, pp. 1-11, Article ID 1559325820936189, 2020.

[102] R. Schmitz, "Friedrich Wilhelm SertÜrner and the discovery of morphine," Pharmaceutical Historian, vol. 27, no. 2, pp. 61-74, 1985.

[103] A. Thawabteh, S. Juma, M. Bader et al., "The biological activity of natural alkaloids against herbivores, cancerous cells and pathogens," Toxins, vol. 11, no. 11, p. 656, 2019.

[104] T. P. T. Cushnie, B. Cushnie, and A. J. Lamb, "Alkaloids: an overview of their antibacterial, antibiotic-enhancing and antivirulence activities," International Journal of Antimicrobial Agents, vol. 44, no. 5, pp. 377-386, 2014.

[105] H. S. Cho, S. H. Chang, Y. S. Chung et al., "Synergistic effect of ERK inhibition on tetrandrine-induced apoptosis in A549 human lung carcinoma cells," Journal of Veterinary Science, vol. 10, no. 1, pp. 23-28, 2009.

[106] I. Mackraj, T. Govender, and P. Gathiram, "Sanguinarine," Cardiovascular Drug Reviews, vol. 26, no. 1, pp. 75-83, 2008.

[107] R. Hamoud and J. R. Wink, "Synergistic antimicrobial activity of combinations of sanguinarine and EDTA with vancomycin against multidrug resistant bacteria," Drug Metabolism Letters, vol. 8, pp. 119-128, 2014.

[108] R. X. Yinan Yin, "Berberine: a medicinal compound for the treatment of bacterial infections," Clinical Microbiology: Open Access, vol. 3, no. 3, pp. 1-4, 2014.

[109] M. Chu, M. B. Zhang, Y. C Liu et al., "Role of berberine in the treatment of methicillin-resistant Staphylococcus aureus infections," Scientific Reports, vol. 6, pp. 1-9, Article ID 24748, 2016.

[110] K. Patel, R. B. Singh, and D. K. Patel, "Medicinal significance, pharmacological activities, and analytical aspects of solasodine: a concise report of current scientific literature," Journal of Acute Disease, vol. 2, no. 2, pp. 92-98, 2013.

[111] K. Murugan, "Solanum alkaloids and their pharmaceutical roles: a review," Journal of Analytical \& Pharmaceutical Research, vol. 3, no. 6, pp. 1-14, 2015.

[112] K. Poole, "Pseudomonas aeruginosa: resistance to the max," Frontiers in Microbiology, vol. 2, p. 65, 2011.

[113] P. D. Lister, D. J. Wolter, and N. D. Hanson, "Antibacterialresistant Pseudomonas aeruginosa: clinical impact and complex regulation of chromosomally encoded resistance mechanisms," Clinical Microbiology Reviews, vol. 22, no. 4, pp. 582-610, 2009.

[114] T. Siriyong, S. Chusri, P. Srimanote, V. Tipmanee, and S. P. Voravuthikunchai, "Holarrhena antidysenterica extract and its steroidal alkaloid, conessine, as resistance-modifying agents against extensively drug-resistant Acinetobacter baumannii," Microbial Drug Resistance, vol. 22, no. 4, pp. 273-282, 2016.

[115] V. M. Dembitsky, "Astonishing diversity of natural surfactants: 6. biologically active marine and terrestrial alkaloid glycosides," Lipids, vol. 40, no. 11, pp. 1081-1105, 2005.

[116] S. Zielinska, M. Wójciak-Kosior, M. Dziagwa-Becker et al., "The activity of isoquinoline alkaloids and extracts from Chelidonium majus against pathogenic bacteria and Candida sp," Toxins (Basel), vol. 11, no. 7, pp. 1-13, 2019.

[117] T. K. Beuria, M. K. Santra, and D. Panda, "Sanguinarine blocks cytokinesis in bacteria by inhibiting FtsZ assembly and bundling $\dagger$," Biochemistry, vol. 44, no. 50, pp. 16584-16593, 2005.

[118] B. W Obiang-Obounou, O.-H. Kang, J.-G. Choi et al., "The mechanism of action of sanguinarine against methicillinresistant Staphylococcus aureus," The Journal of Toxicological Sciences, vol. 36, no. 3, pp. 277-283, 2011.

[119] Q. Zhang, Y. Lyu, J. Huang et al., "Antibacterial activity and mechanism of sanguinarine against Providencia rettgeri in vitro," PeerJ, vol. 8, Article ID e9543, 2020.

[120] R. Hamoud, J. Reichling, and M. Wink, "Synergistic antibacterial activity of the combination of the alkaloid sanguinarine with EDTA and the antibiotic streptomycin against multidrug resistant bacteria," Journal of Pharmacy and Pharmacology, vol. 67, no. 2, pp. 264-273, 2015.

[121] R. Wojtyczka, A. Dziedzic, M. Kępa et al., "Berberine enhances the antibacterial activity of selected antibiotics against coagulase-negative Staphylococcus strains in vitro," Molecules, vol. 19, no. 5, pp. 6583-6596, 2014.

[122] X. Zhang, X. Sun, J. Wu et al., "Berberine damages the cell surface of methicillin-resistant Staphylococcus aureus," Frontiers in Microbiology, vol. 11, p. 621, 2020.

[123] M. L. Boulet, C. Isabelle, I. Guay et al., "Tomatidine is a lead antibiotic molecule that targets Staphylococcus aureus ATP Synthase subunit C," Antimicrobial Agents and Chemotherapy, vol. 62, no. 6, 2018.

[124] R. Soltani, H. Fazeli, R. Bahri Najafi, and A. Jelokhanian, "Evaluation of the synergistic effect of tomatidine with several antibiotics against standard and clinical isolates of Staphylococcus aureus, Enterococcus faecalis, Pseudomonas aeruginosa and Escherichia coli," Iranian Journal of Pharmaceutical Research: IJPR, vol. 16, no. 1, pp. 290-296, 2017.

[125] T. Siriyong, S. P. Voravuthikunchai, and P. J. Coote, "Steroidal alkaloids and conessine from the medicinal plant Holarrhena antidysenterica restore antibiotic efficacy in a Galleria mellonella model of multidrug-resistant Pseudomonas aeruginosa infection $11 \mathrm{Medical}$ and Health Sciences 1108 Medical Microbiolo," BMC Complementary and Alternative Medicine, vol. 18, no. 1, pp. 1-10, 2018.

[126] N. Kumar, B. Singh, P. Bhandari, A. P. Gupta, and V. K. Kaul, "Steroidal alkaloids from Holarrhena antidysenterica (L.) WALL," Chemical and Pharmaceutical Bulletin, vol. 55, no. 6, pp. 912-914, 2007.

[127] M. Laws, A. Shaaban, and K. M. Rahman, "Antibiotic resistance breakers: current approaches and future directions," FEMS Microbiology Reviews, vol. 43, no. 5, pp. 490-516, 2019.

[128] C. Hochfellner, D. Evangelopoulos, M. Zloh et al., “Antagonistic effects of indoloquinazoline alkaloids on antimycobacterial activity of evocarpine," Journal of Applied Microbiology, vol. 118, no. 4, pp. 864-872, 2015.

[129] V. Kuete, J. D. Wansi, A. T. Mbaveng et al., “Antimicrobial activity of the methanolic extract and compounds from Teclea afzelii (Rutaceae)," South African Journal of Botany, vol. 74, no. 4, pp. 572-576, 2008.

[130] G. R. Dwivedi, A. Maurya, D. K. Yadav et al., "Synergy of clavine alkaloid 'chanoclavine' with tetracycline against multi-drug-resistant E. coli," Journal of Biomolecular Structure and Dynamics, vol. 37, no. 5, pp. 1307-1325, 2019.

[131] D. Mabhiza, T. Chitemerere, and S. Mukanganyama, "Antibacterial properties of alkaloid extracts from Callistemon citrinus and Vernonia adoensis against Staphylococcus aureus and Pseudomonas aeruginosa," International Journal of Medicinal Chemistry, vol. 2016, Article ID 6304163, 7 pages, 2016. 
[132] R. S. Costa, M. O. Lins, M. Le Hyaric, T. F. Barros, and E. S. Velozo, "In vitro antibacterial effects of Zanthoxylum tingoassuiba root bark extracts and two of its alkaloids against multiresistant Staphylococcus aureus," Revista Brasileira de Farmacognosia, vol. 27, no. 2, pp. 195-197, 2017.

[133] A. Ismaili, S. Sohrabi, M. Azadpour, R. Heydari, and M. Rashidipour, "Evaluation of the antimicrobial activity of alkaloid extracts of four papaver species," Journal of Herbal Medicine, vol. 2, 2017.

[134] L. Othman, A. Sleiman, and R. M. Abdel-Massih, "Antimicrobial activity of polyphenols and alkaloids in middle eastern plants," Frontiers in Microbiology, vol. 10, p. 911, 2019.

[135] N. Malik and S. Ahmed, "Antimicrobial activity of Carica Papaya,Piper nigrum and Datura stramonium plants on drug resistant pathogens isolated from clinical specimens," IOSR Journal of Biotechnology and Biochemistry, vol. 2, no. 6, pp. 56-61, 2016.

[136] M. S. Aslam, M. S. Ahmad, M. A. Ahmad, and M. Akhlaq, "An updated review on phytochemical and pharmacological propeties of Piper sarmentosum," Current Trends in Biotechnology and Pharmacy, vol. 11, no. 4, pp. 345-356, 2017.

[137] D. Lin, M. Xiao, J Zhao et al., "An overview of plant phenolic compounds and their importance in human nutrition and management of type 2 diabetes," Molecules (Basel, Switzerland), vol. 21, no. 10, 2016.

[138] K. Ganesan and B. Xu, "A critical review on polyphenols and health benefits of black soybeans," Nutrients, vol. 9, no. 5, pp. 1-17, 2017.

[139] M. Abbas, F. Saeed, F. M. Anjum et al., "Natural polyphenols: an overview," International Journal of Food Properties, vol. 20, no. 8, pp. 1689-1699, 2017.

[140] M. Daglia, "Polyphenols as antimicrobial agents," Current Opinion in Biotechnology, vol. 23, no. 2, pp. 174-181, 2012.

[141] Y. Yuheng and T. Zhang, "Antimicrobial activities of tea polyphenol on phytopathogens: a review," Molecules, vol. 24, p. 816, 2019.

[142] F. Farhadi, B. Khameneh, M. Iranshahi, and M. Iranshahy, "Antibacterial activity of flavonoids and their structure-activity relationship: an update review," Phytotherapy Research, vol. 33, no. 1, pp. 13-40, 2019.

[143] S. Siriwong, Y. Teethaisong, K. Thumanu, B. Dunkhunthod, and G. Eumkeb, "The synergy and mode of action of quercetin plus amoxicillin against amoxicillin-resistant Staphylococcus epidermidis," BMC Pharmacology \& Toxicology, vol. 17, no. 1, pp. 39-14, 2016.

[144] C. Vipin, K. Saptami, F. Fida et al., "Potential synergistic activity of quercetin with antibiotics against multidrug-resistant clinical strains of Pseudomonas aeruginosa," PLoS One, vol. 15, no. 11, Article ID e0241304, 2020.

[145] S. Qu, C. Dai, Z. Shen et al., "Mechanism of synergy between tetracycline and quercetin against antibiotic resistant Escherichia coli," Frontiers in Microbiology, vol. 10, p. 2536, 2019.

[146] S. Ndendoung, J. D. D. Tamokou, L. Havyarimana et al., "Antimicrobial and antioxidant activity of kaempferol rhamnoside derivatives from Bryophyllum pinnatum," BMC Research Notes, vol. 5, p. 158, 2012.

[147] J. Shao, M. Zhang, T. Wang, Y. Li, and C. Wang, "The roles of CDR1,CDR2, and MDR1 in kaempferol-induced suppression with fluconazole-resistant Candida albicans," Pharmaceutical Biology, vol. 54, no. 6, pp. 984-992, 2016.

[148] J. G. Holler, S. B. Christensen, H.-C. Slotved et al., "Novel inhibitory activity of the Staphylococcus aureus NorA efflux pump by a kaempferol rhamnoside isolated from Persea lingue Nees," Journal of Antimicrobial Chemotherapy, vol. 67, no. 5, pp. 1138-1144, 2012.

[149] O. Prakash, R. Singh, N. Singh et al., "Exploring the potentials of quercetin and kaempferol combinations along with regular antibiotics for the effective management of methicillin-resistant Staphylococcus aureus (MRSA)," Research \& Reviews: A Journal of Microbiology \& Virology, vol. 8, no. 3, pp. 6-9, 2018.

[150] B. Garg and C. Maliye, "Adolescent health and adolescent health programs in India," Journal of Mahatma Gandhi Institute of Medical Sciences, vol. 22, no. 2, pp. 78-22, 2017.

[151] K. Giri, B. K. Shrestha, J. Shakya, S. N. Sah, and H. Khanal, "Antibacterial effect of green tea extract against multi drug resistant Escherichia coli isolated from urine sample of patients visiting tertiary care hospital of Eastern Nepal," International Journal of Applied Sciences and Biotechnology, vol. 8, no. 1, pp. 45-51, 2020.

[152] J. Gopal, M. Muthu, D. Paul, D. H. Kim, and S. Chun, "Bactericidal activity of green tea extracts: the importance of catechin containing nano particles," Scientific Reports, vol. 6, pp. 1-14, Article ID 19710, 2016.

[153] S. Archana and J. Abraham, "Comparative analysis of antimicrobial activity of leaf extracts from fresh green tea, commercial green tea and black tea on pathogens," Journal of Applied Pharmaceutical Science, vol. 1, no. 8, pp. 152-149, 2011.

[154] H. Y. Mahmood, S. Jamshidi, J. M. Sutton, and K. M. Rahman, "Current advances in developing inhibitors of bacterial multidrug efflux pumps," Current Medicinal Chemistry, vol. 23, no. 10, pp. 1062-1081, 2016.

[155] M. J. Matos, S. Vazquez-Rodriguez, E. Uriarte, and L. Santana, "Potential pharmacological uses of chalcones: a patent review (from June 2011-2014)," Expert Opinion on Therapeutic Patents, vol. 25, no. 3, pp. 351-366, 2015.

[156] G. Belofsky, D. Percivill, K. Lewis, G. P. Tegos, and J. Ekart, "Phenolic metabolites of Daleaversi color that enhance antibiotic activity against model pathogenic bacteria," Journal of Natural Products, vol. 67, no. 3, pp. 481-484, 2004.

[157] H. Koudokpon, N. Armstrong, T. V. Dougnon et al., "Antibacterial activity of chalcone and dihydrochalcone compounds from Uvaria chamae roots against multidrugresistant bacteria," BioMed Research International, vol. 2018, Article ID 1453173, 10 pages, 2018.

[158] G.-S. Lee, E.-S. Kim, S.-I. Cho et al., "Antibacterial and synergistic activity of prenylated chalcone isolated from the roots of Sophora flavescens," Journal of the Korean Society for Applied Biological Chemistry, vol. 53, no. 3, pp. 290-296, 2010.

[159] C. Babii, L. G. Bahrin, A.-N. Neagu et al., "Antibacterial activity and proposed action mechanism of a new class of synthetic tricyclic flavonoids," Journal of Applied Microbiology, vol. 120, no. 3, pp. 630-637, 2016.

[160] Y. Prasad, L. R. Atmakuri, and R. Rambabu, "Synthesis and antimicrobial activity of some chalcone derivatives," Journal of Chemistry, vol. 5, Article ID 876257, 6 pages, 2008.

[161] M. S. Alam, S. M. M. Rahman, and D. U. Lee, "Synthesis, biological evaluation, quantitative-SAR and docking studies of novel chalcone derivatives as antibacterial and antioxidant agents," Chemical Papers, vol. 69, no. 8, pp. 1118-29, 2015.

[162] H. Edziri, M. Mastouri, M. A. Mahjoub, Z. Mighri, A. Mahjoub, and L. Verschaeve, "Antibacterial, antifungal and cytotoxic activities of two flavonoids from Retama 
raetam flowers," Molecules (Basel, Switzerland), vol. 17, no. 6, pp. 7284-7293, 2012.

[163] M. Fujita, S. Shiota, T. Kuroda et al., "Remarkable synergies between baicalein and tetracycline, and baicalein and $\beta$-lactams against methicillin-resistant Staphylococcus aureus," Microbiology and Immunology, vol. 49, no. 4, pp. 391-396, 2005.

[164] B. C. L. Chan, M. Ip, C. B. S. Lau et al., "Synergistic effects of baicalein with ciprofloxacin against NorA over-expressed methicillin-resistant Staphylococcus aureus (MRSA) and inhibition of MRSA pyruvate kinase," Journal of Ethnopharmacology, vol. 137, no. 1, pp. 767-773, 2011.

[165] Y. S. L. Eun Kyung Jung, "Synergistic effect between baicalein and antibiotics against clinic methicillin and vancomycin-resistant Staphylococcus aureus," Chemother Open Access, vol. 4, no. 1, pp. 1-11, 2014.

[166] M. Vestergaard and H. Ingmer, "Antibacterial and antifungal properties of resveratrol," International Journal of Antimicrobial Agents, vol. 53, no. 6, pp. 716-723, 2019.

[167] L. Paulo, S. Ferreira, E. Gallardo, J. A. Queiroz, and F. Domingues, "Antimicrobial activity and effects of resveratrol on human pathogenic bacteria," World Journal of Microbiology and Biotechnology, vol. 26, no. 8, pp. 15331538, 2010.

[168] S. Kanakarajan and R. Selvaraj, "Evaluation of the in vitro antimicrobial effect of resveratrol on human pathogens," International Journal of Zoology Studies, vol. 2, 2017.

[169] A. Klančnik, M. Šikić Pogačar, K. Trošt, M. Tušek Žnidarič, B. Mozetič Vodopivec, and S. Smole Možina, "Anti-campylobacter activity of resveratrol and an extract from waste Pinot noir grape skins and seeds, and resistance of Camp. jejuni planktonic and biofilm cells, mediated via the CmeABC efflux pump," Journal of Applied Microbiology, vol. 122, no. 1, pp. 65-77, 2017.

[170] T. Khan, K. Sankhe, V. Suvarna, A. Sherje, K. Patel, and B. Dravyakar, "DNA gyrase inhibitors: progress and synthesis of potent compounds as antibacterial agents," Biomedicine \& Pharmacotherapy, vol. 103, pp. 923-938, 2018.

[171] K. Patel, C. Tyagi, S. Goyal et al., "Identification of chebulinic acid as potent natural inhibitor of M. tuberculosis DNA gyrase and molecular insights into its binding mode of action," Computational Biology and Chemistry, vol. 59, pp. 37-47, 2015.

[172] Y. Miyasaki, J. D. Rabenstein, J. Rhea et al., "Isolation and characterization of antimicrobial compounds in plant extracts against multidrug-resistant acinetobacter baumannii," PLoS One, vol. 8, no. 4, Article ID e61594, 2013.

[173] K. B. Myint, L. C. Sing, and Z. Wei, "Tannic acid as phytochemical potentiator for antibiotic resistance adaptation," APCBEE Procedia, vol. 7, pp. 175-181, 2013.

[174] A. Borges, C. Ferreira, M. J. Saavedra, and M. Simões, "Antibacterial activity and mode of action of ferulic and gallic acids against pathogenic bacteria," Microbial Drug Resistance, vol. 19, no. 4, pp. 256-265, 2013.

[175] G. Kim, R. Y. Gan, D. Zhang et al., "Large-scale screening of 239 traditional Chinese medicinal plant extracts for their antibacterial activities against multidrug-resistant Staphylococcus aureus and cytotoxic activities," Pathogens (Basel, Switzerland), vol. 9, no. 3, 2020.

[176] R. Barbieri, E. Coppo, A. Marchese et al., "Phytochemicals for human disease: an update on plant-derived compounds antibacterial activity," Microbiological Research, vol. 196, pp. 44-68, 2017.
[177] M. Nakamoto, K. Kunimura, J. Suzuki, and Y. Kodera, "Antimicrobial properties of hydrophobic compounds in garlic: allicin, vinyldithiin, ajoene and diallyl polysulfides (review)," Experimental and Therapeutic Medicine, vol. 19, no. 2, pp. 1550-1553, 2019.

[178] V. Lanzotti, F. Scala, and G. Bonanomi, "Compounds from Allium species with cytotoxic and antimicrobial activity," Phytochemistry Reviews, vol. 13, no. 4, pp. 769-791, 2014.

[179] M. A. Farag, S. E. Ali, R. H. Hodaya et al., "Phytochemical profiles and antimicrobial activities of Allium cepa red cv. and $A$. sativum subjected to different drying methods: a comparative MS-based metabolomics," Molecules (Basel, Switzerland), vol. 22, no. 5, 2017.

[180] J. Reiter, A. M. Hübbers, F. Albrecht, L. I. O. Leichert, and A. J. Slusarenko, "Allicin, a natural antimicrobial defence substance from garlic, inhibits DNA gyrase activity in bacteria," International Journal of Medical Microbiology, vol. 310, no. 1, Article ID 151359, 2020.

[181] C. J. Cavallito and J. H. Bailey, "Allicin, the antibacterial principle of Allium sativum. I. Isolation, physical properties and antibacterial action," Journal of the American Chemical Society, vol. 66, no. 11, pp. 1950-1951, 1944.

[182] R. Leontiev, N. Hohaus, C. Jacob, M. C. H. Gruhlke, and A. J. Slusarenko, "A comparison of the antibacterial and antifungal activities of thiosulfinate analogues of allicin," Scientific Reports, vol. 8, no. 1, p. 6763, 2018.

[183] S. Montaut, J. Barillari, R. Iori, and P. Rollin, "Glucoraphasatin: chemistry, occurrence, and biological properties," Phytochemistry, vol. 71, no. 1, pp. 6-12, 2010.

[184] L. Romeo, R. Iori, P. Rollin, P. Bramanti, and E. Mazzon, "Isothiocyanates: an overview of their antimicrobial activity against human infections," Molecules (Basel, Switzerland), vol. 23, no. 3, pp. 1-18, 2018.

[185] Z. Lu, C. R. Dockery, M. Crosby, K. Chavarria, B. Patterson, and M. Giedd, "Antibacterial activities of wasabi against Escherichia coli O157:H7 and Staphylococcus aureus," Frontiers in Microbiology, vol. 7, p. 1403, 2016.

[186] C.-M. Lin, J. F. PrestonIII, and C.-I. Wei, “Antibacterial mechanism of allyl isothiocyanate," Journal of Food Protection, vol. 63, no. 6, pp. 727-734, 2000.

[187] F. B. Luciano and R. A. Holley, "Enzymatic inhibition by allyl isothiocyanate and factors affecting its antimicrobial action against Escherichia coli O157:H7," International Journal of Food Microbiology, vol. 131, no. 2, pp. 240-245, 2009.

[188] S. J. Kaiser, N. T. Mutters, B. Blessing, and F. Günther, "Natural isothiocyanates express antimicrobial activity against developing and mature biofilms of Pseudomonas aeruginosa," Fitoterapia, vol. 119, pp. 57-63, 2017.

[189] A. AbreuA., A. Borges, L. Simoes, M. Saavedra, and M. Simoes, "Antibacterial activity of phenyl isothiocyanate on Escherichia coli and Staphylococcus aureus," Medicinal Chemistry, vol. 9, no. 5, pp. 756-761, 2013.

[190] C. Dias, A. Aires, and M. Saavedra, "Antimicrobial activity of isothiocyanates from cruciferous plants against methicillinresistant Staphylococcus aureus (MRSA)," International Journal of Molecular Sciences, vol. 15, no. 11, pp. 1955219561, 2014.

[191] A. Sofrata, E. M. Santangelo, M. Azeem, A.-K. Borg-Karlson, A. Gustafsson, and K. Pütsep, "Benzyl isothiocyanate, a major component from the roots of Salvadora persica is highly active against gram-negative bacteria," PLoS One, vol. 6, no. 8, Article ID e23045, 2011. 
[192] J.-A. Choi, S.-N. Cho, Y.-J. Lim et al., "Enhancement of the antimycobacterial activity of macrophages by ajoene," Innate Immunity, vol. 24, no. 1, pp. 79-88, 2018.

[193] C. H. Kaschula, R. Hunter, and M. I. Parker, "Garlic-derived anticancer agents: structure and biological activity of ajoene," BioFactors (Oxford, England), vol. 36, no. 1, pp. 78-85, 2010.

[194] T. H. Jakobsen, M. van Gennip, R. K. Phipps et al., “Ajoene, a sulfur-rich molecule from garlic, inhibits genes controlled by quorum sensing," Antimicrobial Agents and Chemotherapy, vol. 56, no. 5, pp. 2314-2325, 2012.

[195] J. Lei, L. Sun, S. Huang et al., "The antimicrobial peptides and their potential clinical applications," American Journal of Translational Research, vol. 11, no. 7, pp. 3919-3931, 2019.

[196] B. R. Samriti and K. Biswas, "Antibacterial activity of antimicrobial peptide extracted from Trianthema portulacastrum leaves," Journal of Pharmaceutical Innovation-SCI Journal, vol. 8, no. 3, pp. 81-86, 2019.

[197] R. Nawrot, J. Barylski, G. Nowicki, J. Broniarczyk, W. Buchwald, and A. Goździcka-Józefiak, "Plant antimicrobial peptides," Folia Microbiologica, vol. 59, no. 3, pp. 181-196, 2014.

[198] T. Cangiano, M. DellaGreca, A. Fiorentino, M. Isidori, P. Monaco, and A. Zarrelli, "Lactone diterpenes from the aquatic plant Potamogeton natans," Phytochemistry, vol. 56, no. 5, pp. 469-473, 2001.

[199] N. A. Mahizan, S. Yang, C.-L. Moo, and A. A.-L. Song, "Terpene derivatives as a potential agent against," Molecules, vol. 24, no. 2631, pp. 1-21, 2019.

[200] S. A. Zacchino, E. Butassi, E. Cordisco, and L. A. Svetaz, "Hybrid combinations containing natural products and antimicrobial drugs that interfere with bacterial and fungal biofilms," Phytomedicine, vol. 37, pp. 14-26, 2017.

[201] B. Singh and R. A. Sharma, "Plant terpenes: defense responses, phylogenetic analysis, regulation and clinical applications," 3 Biotech, vol. 5, no. 2, pp. 129-151, 2015.

[202] M. E. Bergman, B. Davis, and M. A. Phillips, "Medically useful plant terpenoids: biosynthesis, occurrence, and mechanism of action," Molecules, vol. 24, no. 21, p. 3961, 2019.

[203] Y. El Atki, I. Aouam, F. El Kamari et al., "Antibacterial efficacy of thymol, carvacrol, eugenol and menthol as alternative agents to control the growth of nosocomial infection-bacteria," Journal of Pharmaceutical Sciences and Research, vol. 11, no. 2, pp. 306-309, 2019.

[204] A. Marchese, C. R. Arciola, R. Barbieri et al., "Update on monoterpenes as antimicrobial agents: a particular focus on p-cymene," Materials (Basel, Switzerland), vol. 10, no. 8, pp. 1-15, 2017.

[205] V. Lorenzi, A. Muselli, A. F. Bernardini et al., "Geraniol restores antibiotic activities against multidrug-resistant isolates from gram-negative species," Antimicrobial Agents and Chemotherapy, vol. 53, no. 5, pp. 2209-2211, 2009.

[206] M. Cristani, M. D’Arrigo, G. Mandalari et al., "Interaction of four monoterpenes contained in essential oils with model membranes: implications for their antibacterial activity," Journal of Agricultural and Food Chemistry, vol. 55, no. 15, pp. 6300-6308, 2007.

[207] S. García-Salinas, H. Elizondo-Castillo, M. Arruebo, G. Mendoza, and S. Irusta, "Evaluation of the antimicrobial activity and cytotoxicity of different components of natural origin present in essential oils," Molecules, vol. 23, no. 6, p. 1399, 2018.
[208] R. Chaudhuri and K. Bojanowski, "Bakuchiol: a retinol-like functional compound revealed by gene expression profiling \& clinically proven to have anti-aging effects," International Journal of Cosmetic Science, vol. 36, no. 3, pp. 221-230, 2014.

[209] A. N. Feneran, W. S. Kaufman, T. S. Dabade, and S. R. Feldman, "Retinoid plus antimicrobial combination treatments for acne," Clinical, Cosmetic and Investigational Dermatology, vol. 4, pp. 79-92, 2011.

[210] M. C. Liggins, F. Li, L.-J. Zhang, T. Dokoshi, and R. L. Gallo, "Retinoids enhance the expression of cathelicidin antimicrobial peptide during reactive dermal adipogenesis," The Journal of Immunology, vol. 203, no. 6, pp. 1589-1597, 2019.

[211] L. Beckenbach, J. M. Baron, H. F. Merk, H. Löffler, and P. M. Amann, "Retinoid treatment of skin diseases," European Journal of Dermatology, vol. 25, no. 5, pp. 384-391, 2015.

[212] F. Tan, P. She, L. Zhou et al., "Bactericidal and anti-biofilm activity of the retinoid compound CD437 against Enterococcus faecalis," Frontiers in Microbiology, vol. 10, p. 2301, 2019.

[213] W. Kim, W. Zhu, G. L. Hendricks et al., "A new class of synthetic retinoid antibiotics effective against bacterial persisters," Nature, vol. 556, pp. 103-107, 2018.

[214] J. A. Jesus, J. H. G. Lago, M. D. Laurenti, E. S. Yamamoto, and L. F. D. Passero, "Antimicrobial activity of oleanolic and ursolic acids: an update," Evidence-Based Complementary and Alternative Medicine, vol. 2015, Article ID 620472, 14 pages, 2015.

[215] R. T. Nzogong, F. S. T. Ndjateu, S. E. Ekom et al., "Antimicrobial and antioxidant activities of triterpenoid and phenolic derivatives from two cameroonian melastomataceae plants: Dissotis senegambiensis and Amphiblemma monticola," BMC Complementary and Alternative Medicine, vol. 18, no. 1, p. 159, 2018.

[216] T. B. Ayeleso, M. G. Matumba, and E. Mukwevho, "Oleanolic acid and its derivatives: biological activities and therapeutic potential in chronic diseases," Molecules, vol. 22, no. 11, 2017.

[217] K. Horiuchi, S. Shiota, T. Hatano, T. Yoshida, T. Kuroda, and T. Tsuchiya, "Antimicrobial activity of oleanolic acid from Salvia officinalis and related compounds on vancomycinresistant enterococci (VRE)," Biological and Pharmaceutical Bulletin, vol. 30, no. 6, pp. 1147-1149, 2007.

[218] S. Fontanay, M. Grare, J. Mayer, C. Finance, and R. E. Duval, "Ursolic, oleanolic and betulinic acids: antibacterial spectra and selectivity indexes," Journal of Ethnopharmacology, vol. 120 , no. 2 , pp. $272-276,2008$

[219] S.-G. Kim, M.-J. Kim, D.-C. Jin et al., "Antimicrobial effect of ursolic acid and oleanolic acid against methicillin-resistant Staphylococcus aureus," The Korean Journal of Microbiology, vol. 48, no. 3, pp. 212-215, 2012.

[220] A. Kurek, K. Markowska, A. M. Grudniak, W. Janiszowska, and K. I. Wolska, "The effect of oleanolic and ursolic acids on the hemolytic properties and biofilm formation of Listeria monocytogenes," Polish Journal of Microbiology, vol. 63, no. 1, pp. 21-25, 2014.

[221] T. Zhou, Z. Li, O.-H. Kang et al., "Antimicrobial activity and synergism of ursolic acid 3-O- $\alpha$-L-arabinopyranoside with oxacillin against methicillin-resistant Staphylococcus aureus," International Journal of Molecular Medicine, vol. 40, no. 4, pp. 1285-1293, 2017.

[222] R. Sathasivam and J.-S. Ki, "A review of the biological activities of microalgal carotenoids and their potential use in 
healthcare and cosmetic industries," Marine Drugs, vol. 16, no. 1, p. 26, 2018.

[223] T. M. Keceli, Z. Erginkaya, E. Turkkan, and U. Kaya, "Antioxidant and antibacterial effects of carotenoids extracted from Rhodotorula glutinis strains," Asian Journal of Chemistry, vol. 25, no. 1, pp. 42-46, 2013.

[224] T. M. Karpiński and A. Adamczak, "Fucoxanthin-an antibacterial carotenoid," Antioxidants, vol. 8, no. 8, pp. 1-8, 2019.

[225] M. Ghavam, M. L. Manca, M. Manconi, and G. Bacchetta, "Chemical composition and antimicrobial activity of essential oils obtained from leaves and flowers of Salvia hydrangea DC. ex benth," Scientific Reports, vol. 10, no. 1, pp. 1-10, 2020.

[226] K. N. Venugopala, V. Rashmi, and B. Odhav, "Review on natural coumarin lead compounds for their pharmacological activity," BioMed Research International, vol. 2013, Article ID 963248, 14 pages, 2013.

[227] R. Pati and M. Muthukumar, "Genetic transformation of bael (Aegle marmelos corr.)," in Biotechnology of Neglected and Underutilized Cropspp. 343-365, 1st edition, 2013.

[228] I. Kostova, S. Raleva, P. Genova, and R. Argirova, "Recent advances in the discovery and development of plant-derived natural coumarins and their analogues as anti human immunodeficiency virus-type 1 (HIV-1) agents," Biotechnology \& Biotechnological Equipment, vol. 19, no. 1, pp. 16-22, 2005.

[229] A. Basile, S. Sorbo, V. Spadaro et al., "Antimicrobial and antioxidant activities of coumarins from the roots of Ferulago campestris (apiaceae)," Molecules, vol. 14, no. 3, pp. 939-952, 2009.

[230] S. S. S. A. Aziz, M. A. Sukari, M. Rahmani, M. Kitajima, N. Aimi, and N. J. Ahpandi, "Koumarin daripada Murraya paniculata (rutaceae)," The Malaysian Journal of Analytical Sciences, vol. 14, no. 1, pp. 1-5, 2010.

[231] Shakeel-U-Rehman, R. Khan, K. A. Bhat, A. F. Raja, A. S. Shawl, and M. S. Alam, "Isolation, characterisation and antibacterial activity studies of coumarins from Rhododendron lepidotum wall. ex g. don, ericaceae," Revista Brasileira de Farmacognosia, vol. 20, no. 6, pp. 886-890, 2010.

[232] D. Dastan, P. Salehi, A. Aliahmadi, A. R. Gohari, H. Maroofi, and A. Ardalan, "New coumarin derivatives from Ferula pseudalliacea with antibacterial activity," Natural Product Research, vol. 30, no. 24, pp. 2747-2753, 2016.

[233] H. R. El-Seedi, "Antimicrobial arylcoumarins from Asphodelus microcarpus," Journal of Natural Products, vol. 70, no. 1, pp. 118-120, 2007.

[234] O. Kayser and H. Kolodziej, "Antibacterial activity of simple coumarins: structural requirements for biological activity," Zeitschrift für Naturforschung C, vol. 54, no. 3-4, pp. 169-174, 1999.

[235] A. Maxwell, "The interaction between coumarin drugs and DNA gyrase," Molecular Microbiology, vol. 9, no. 4, pp. 681-686, 1993.

[236] L. da S.M. Forezi, T. Q. Froes, M. F. C. Cardoso et al., "Synthesis and biological evaluation of coumarins derivatives as potential inhibitors of the production of Pseudomonas aeruginosa virulence factor pyocyanin," Current Topics in Medicinal Chemistry, vol. 18, no. 2, pp. 149-156, 2018.

[237] G. Martelli and D. Giacomini, "Antibacterial and antioxidant activities for natural and synthetic dual-active compounds," European Journal of Medicinal Chemistry, vol. 158, pp. 91$105,2018$.
[238] S. Y. Kim, C. Park, H.-J. Jang et al., "Antibacterial strategies inspired by the oxidative stress and response networks," Journal of Microbiology, vol. 57, no. 3, pp. 203-212, 2019.

[239] M. B. Pisano, A. Kumar, R Medda et al., "Antibacterial activity and molecular docking studies of a selected series of hydroxy-3-arylcoumarins," Molecules (Basel, Switzerland), vol. 24 , no. 15 , pp. 1-14, 2019.

[240] A. V. Lipeeva, D. O. Zakharov, L. G. Burova et al., "Design, synthesis and antibacterial activity of coumarin-1,2,3-triazole hybrids obtained from natural furocoumarin peucedanin," Molecules (Basel, Switzerland), vol. 24, no. 11, 2019.

[241] J. Sahoo and S. K. Paidesetty, "Antimicrobial activity of novel synthesized coumarin based transitional metal complexes," Journal of Taibah University Medical Sciences, vol. 12, no. 2, pp. 115-124, 2017.

[242] J. Sahoo and S. K. Paidesetty, "Biological investigation of novel metal complexes of 2-amino-4-substituted phenylthiazole Schiff bases," Journal of Taibah University Medical Sciences, vol. 13, no. 2, pp. 142-155, 2018.

[243] K. Bhagat, J. Bhagat, M. K. Gupta et al., "Design, synthesis, antimicrobial evaluation, and molecular modeling studies of novel indolinedione-coumarin molecular hybrids," ACS Omega, vol. 4, no. 5, pp. 8720-8730, 2019.

[244] M. I. A. Dobara and A. Z. E. Sonbati, "Thermal properties, antimicrobial activity of azo complexes and ultrastructure study of some affected bacteria," Journal of Microbial and Biochemical Technology, vol. s3, 2011.

[245] D. Qu, Z. Hou, J Li et al., "A new coumarin compound DCH combats methicillin-resistant Staphylococcus aureus biofilm by targeting arginine repressor," Science Advances, vol. 6, no. 30, Article ID eaay9597, 2020. 